\title{
An inhomogeneous Lambda-determinant
}

\author{
Philippe Di Francesco \\ Department of Mathematics \\ University of Illinois MC-382 \\ Urbana, IL 61821, U.S.A. \\ philippe@illinois.edu
}

Submitted: Sep 29, 2012; Accepted: Aug 5, 2013; Published: Aug 16, 2013

Mathematics Subject Classifications: 05C22; 05E10; 05A15; 05A30; 82B20.

\begin{abstract}
We introduce a multi-parameter generalization of the Lambda-determinant of Robbins and Rumsey, based on the cluster algebra with coefficients attached to a $T$-system recurrence. We express the result as a weighted sum over alternating sign matrices.
\end{abstract}

\section{Introduction}

The so-called Lambda-determinant was introduced by Robbins and Rumsey [14] as a natural generalization of the ordinary determinant, via a one-parameter deformation of the Dodgson condensation algorithm [7] that expresses the determinant of any $n \times n$ matrix in terms of minors of sizes $n-1$ and $n-2$. This produces, for each $n \times n$ matrix $A$, a Laurent polynomial of its entries, involving only monomials with powers of \pm 1 , coded by Alternating Sign Matrices (ASM) of same size $n$. Robbins and Rumsey were able to write a compact formula for the Lambda-determinant of any matrix $A$, as a sum over the ASMs of same size with explicit coefficients [14] (see also [2] for a lively account of the discovery of ASMs). ASMs are known to be in bijection with configurations of the Six Vertex $(6 \mathrm{~V})$ model on a square $n \times n$ grid, with special Domain Wall Boundary Conditions (DWBC) [10]. The latter are obtained by choosing an orientation of the edges of the underlying square lattice, such that each vertex of the grid has two incoming and two outgoing adjacent edges. The resulting six local configurations read as follows:

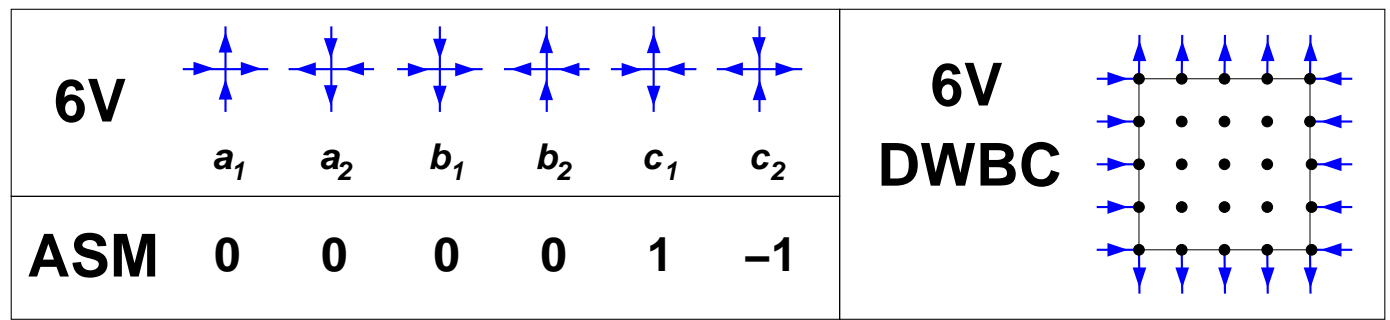


The boundary condition (DWBC) imposes that horizontal external edges point towards the grid, while vertical external edges point out of the grid. The bijection between the $6 \mathrm{~V}$ configurations and ASMs goes as follows: the $a$ and $b$ type vertices correspond to the entries 0 , while the $c_{1}$ type vertex corresponds to the entry 1 , and the $c_{2}$ type vertex to the entry -1 . Conversely, for any $n \times n \operatorname{ASM} B$, there is a unique $6 \mathrm{~V}$-DWBC configuration on the $n \times n$ square grid. For any fixed $\operatorname{ASM} B$, we denote by $\mathcal{C}(B)_{i, j}$ the configuration of the vertex $(i, j)$ in the corresponding $6 \mathrm{~V}$-DWBC model, with $\mathcal{C}(B)_{i, j} \in\left\{a_{1}, a_{2}, b_{1}, b_{2}, c_{1}, c_{2}\right\}$.

In this note, we define an inhomogeneous, multi-parameter generalization of the Lambda-determinant of an $n \times n$ matrix $A$. This is done by deforming the Dodgson condensation algorithm [7] by use of two sets of parameters $\lambda_{a}, \mu_{a}, a \in \mathbb{Z}$. More precisely:

Definition 1. Let $A=\left(a_{i, j}\right)_{i, j \in I}, I=\{1,2, \ldots, n\}$, and sequences $\lambda=\left(\lambda_{a}\right)_{a \in \mathbb{Z}}, \mu=$ $\left(\mu_{a}\right)_{a \in \mathbb{Z}}$ of fixed parameters. The generalized Lambda-determinant of $A$, denoted $|A|_{\lambda, \mu}$ is defined inductively by the following modified Dodgson condensation algorithm. Let $A_{i_{1}, \ldots, i_{k}}^{j_{1}, \ldots, j_{k}}$ denote the submatrix of $A$ obtained by erasing rows $i_{1}, \ldots, i_{k}$ and columns $j_{1}, \ldots, j_{k}$, and $s$ be the shift operator acting on sequences as $(s \lambda)_{a}=\lambda_{a+1}$ and $\left(s^{-1} \lambda\right)_{a}=\lambda_{a-1}$ and similarly on $\mu$. We have:

$$
|A|_{\lambda, \mu}=\frac{\mu_{0}\left|A_{1}^{1}\right|_{s \lambda, s \mu}\left|A_{n}^{n}\right|_{s^{-1} \lambda, s^{-1} \mu}+\lambda_{0}\left|A_{1}^{n}\right|_{s^{-1} \lambda, s \mu}\left|A_{n}^{1}\right|_{s \lambda, s^{-1} \mu}}{\left|A_{1, n}^{1, n}\right|_{\lambda, \mu}}
$$

This recursion relation on the size of the matrix, together with the initial condition that a $0 \times 0$ matrix has generalized Lambda-determinant 1 and $a 1 \times 1$ matrix $(a)$ has generalized Lambda-determinant a determines $|A|_{\lambda, \mu}$ completely.

This generalization of the Lambda-determinant is connected to the expressions found in [15] for solutions of the octahedron recurrence as partition functions of domino tilings of Aztec diamonds. The main result of the present paper is the following closed formula, which holds whenever the definition makes sense:

Theorem 2. For fixed parameters $\lambda_{a}, \mu_{a}, a \in\{-n+2,-n+3, \ldots, n-3, n-2\}$ the generalized Lambda-determinant of a matrix $A=\left(a_{i, j}\right)_{i, j \in I}, I=\{1,2, \ldots, n\}$, is expressed as follows:

$$
|A|_{\lambda, \mu}=\sum_{A S M B} \prod_{i, j=1}^{n} w_{i, j}(A, B ; \lambda, \mu)
$$

where the sum extends over all the $n \times n$ Alternating Sign Matrices $B=\left(b_{i, j}\right)_{i, j \in I}$, and the weights $w_{i, j}(A, B, \lambda ; \mu)$ are defined as:

$$
w_{i, j}(A, B ; \lambda, \mu)=\left(a_{i, j}\right)^{b_{i, j}} \times\left\{\begin{array}{cc}
\lambda_{j-i} & \text { if } \mathcal{C}(B)_{i, j}=a_{1} \\
\mu_{i+j-n-1} & \text { if } \mathcal{C}(B)_{i, j}=b_{1} \\
\left(\lambda_{j-i}+\mu_{i+j-n-1}\right) & \text { if } \mathcal{C}(B)_{i, j}=c_{2} \\
1 & \text { otherwise }
\end{array} .\right.
$$


The paper is organized as follows. In Section 2, we recall Robbins and Rumsey's original definition of the Lambda-determinant, and express it as a solution of the $T$ system/octahedron recurrence with one coefficient. In Section 3, we define the generalized Lambda-determinant as a solution of a $T$-system with inhomogeneous coefficients. The latter is shown to be part of a cluster algebra of infinite rank, and as such to possess the Laurent property. Using the T-system relation, we present a non-homogeneous example leading to simple product formulas for the generalized Lambda-determinant of the matrix with entries $a_{i, j}=1$ for all $i, j$. Section 4 is devoted to the actual computation of the generalized Lambda-determinant, by solving the $T$-system with inhomogeneous coefficients. This is done via a matrix representation of the $T$-system relation that leads to a generalization of the solution of [6]. In Section 5 the solution is rephrased in terms of networks, namely of paths on directed graphs. The latter are finally mapped onto configurations of the $6 \mathrm{~V}$-DWBC model, leading to the proof of Theorem 2. Section 6 is devoted to further properties of the generalized Lambda-determinant, an expression purely in terms of ASMs, some explicit examples, and the complete solution of the $T$-system with inhomogeneous coefficients, and finally some concluding remarks.

Acknowledgments. We thank R. Kedem for many useful discussions, and the referee for very helpful comments. We also thank the Mathematical Science Research Institute in Berkeley, CA and the organizers of the semester "Cluster Algebras" for hospitality while this work was completed. This work is partially supported by the CNRS PICS program.

\section{The classical Lambda-determinant}

\subsection{Desnanot-Jacobi identity and determinant}

Let $I=\{1,2, \ldots, n\}$. For any matrix $M=\left(m_{i, j}\right)_{i, j \in I}$ we use the same notations as in Def.1. For any given $k+1 \times k+1$ matrix $M$, we have the celebrated identity:

$$
|M| \times\left|M_{1, n}^{1, n}\right|=\left|M_{n}^{n}\right| \times\left|M_{1}^{1}\right|-\left|M_{1}^{n}\right| \times\left|M_{n}^{1}\right|
$$

This gives rise to the Dodgson condensation algorithm for effective computing of determinants, as the identity may be used as a closed recursion relation on the size of the matrix, allowing for computing its determinant from the initial data of determinants of matrices of size 0 and 1 (equal respectively to 1 and the single matrix element).

More formally, we may recast the algorithm using the so-called $A_{\infty} T$-system relation:

$$
T_{i, j, k+1} T_{i, j, k-1}=T_{i, j+1, k} T_{i, j-1, k}-T_{i+1, j, k} T_{i-1, j, k}
$$

for any $i, j, k \in \mathbb{Z}$ with fixed parity of $i+j+k$.

Let $A=\left(a_{i, j}\right)_{i, j \in I}$ be a fixed $n \times n$ matrix. Together with the initial data:

$$
\begin{aligned}
& T_{\ell, m, 0}=1 \quad(\ell, m \in \mathbb{Z} ; \ell+m=n \bmod 2) \\
& T_{i, j, 1}=a_{\frac{j-i+n+1}{2}}, \frac{i+j+n+1}{2} \quad(i, j \in \mathbb{Z} ; i+j=n+1 \bmod 2 ;|i|+|j| \leqslant n-1),
\end{aligned}
$$


the solution of the $T$-system (2.2) satisfies:

$$
T_{0,0, n}=\operatorname{det}(A)
$$

\section{$2.2 \quad T$-system with a coefficient and Lambda-determinant}

Robbins and Rumsey have introduced the Lambda-determinant by applying the Dodgson algorithm to the following modified Desnanot-Jacobi identity. Let $\lambda \in \mathbb{C}^{*}$ be a fixed parameter and $A=\left(a_{i, j}\right)_{i, j \in I}$ be a fixed $n \times n$ matrix.

Definition 3. The Lambda-determinant of $A$, denoted $|A|_{\lambda}$ is defined as the solution $|A|_{\lambda}=T_{0,0, n}$ of the following $T$-system with a coefficient

$$
T_{i, j, k+1} T_{i, j, k-1}=T_{i, j+1, k} T_{i, j-1, k}+\lambda T_{i+1, j, k} T_{i-1, j, k}
$$

and subject to the initial conditions (2.3).

Note that for $\lambda=-1$, we recover the usual determinant: $|A|_{-1}=\operatorname{det}(A)$.

Example 4. The generalized Lambda-determinant of a $3 \times 3$ matrix $A$ reads:

$$
\left|\begin{array}{lll}
a & b & c \\
d & e & f \\
g & h & k
\end{array}\right|_{\lambda}=\lambda^{3} c e g+\lambda^{2} c d h+\lambda^{2} b f g+a e k+\lambda b d k+\lambda a f h+\lambda(\lambda+1) \frac{b d f h}{e}
$$

Example 5. The Lambda-determinant of the Vandermonde matrix $A=\left(a_{i}^{j-1}\right)_{i, j \in I}$ reads

$$
|A|_{\lambda}=\prod_{1 \leqslant i<j \leqslant n}\left(a_{j}+\lambda a_{i}\right)
$$

\subsection{The Robbins-Rumsey formula and Alternating Sign Matri- ces}

A remarkable property of the definition of the Lambda-determinant, is that it produces a Laurent polynomial of the matrix elements $a_{i, j}$, which is a sum over monomials with only powers \pm 1 . These powers are coded by $n \times n$ so-called Alternating Sign Matrices (ASM), namely matrices $B=\left(b_{i, j}\right)_{i, j \in I}$ with entries $b_{i, j} \in\{0,1,-1\}$, with non-negative partial row sums: $\sum_{j=1}^{k} b_{i, j} \geqslant 0, k=1,2, \ldots, n-1, i \in I$; and with row sums equal to one: $\sum_{j=1}^{n} b_{i, j}=1, i \in I$. More precisely, Robbins and Rumsey found the following formula for the Lambda-determinant:

$$
|A|_{\lambda}=\sum_{A S M B} \lambda^{\operatorname{Inv}(B)}\left(1+\lambda^{-1}\right)^{\#(-1)_{B}} \prod_{i, j \in I} a_{i, j}^{b_{i, j}}
$$

where $\#(-1)_{B}$ is the total number of entries in $B$ that are equal to -1 , and

$$
\operatorname{Inv}(B)=\sum_{\substack{k<\ell \\ m<p}} b_{k, p} b_{\ell, m}
$$

is the generalized inversion number of $B$. 
Remark 6. Note that for $\lambda=-1$ the sum truncates to only the contribution of ASMs with no -1 entry, which are the permutation matrices $P$, and $\operatorname{Inv}(P)$ is the usual inversion number of the permutation matrix $P$, so that (2.6) reduces to the usual determinant formula.

Remark 7. From eq.(2.6), we see that the Lambda-determinant is well defined for any matrix A with non-vanishing entries. More precisely, as the matrix elements $b_{i, j}=-1$ of ASMs may only occur for $2 \leqslant i, j \leqslant n-1$, the Lambda-determinant is well-defined for any $n \times n$ matrix $A$ such that $a_{i, j} \neq 0$ for all $2 \leqslant i, j \leqslant n-1$.

\section{An inhomogeneous generalization of the Lambda- determinant}

\subsection{T-system and Cluster Algebra}

The unrestricted $A_{\infty} T$-system (2.2) is known to be part of an infinite rank cluster algebra with coefficients [9]. The T-system in this form (with $T_{i, j, k}$ defined only for $i+j+k=1$ mod 2), was shown to be a particular mutation in an infinite rank cluster algebra with coefficients, with cluster variables of the form $\left(T_{i, j, k-1}, T_{i^{\prime}, j^{\prime}, k}\right)_{i, j, i^{\prime}, j^{\prime} \in \mathbb{Z}}$ and non-vanishing fixed coefficients $\left(\lambda_{i}\right)_{i \in \mathbb{Z}}[4]$. It reads:

$$
T_{i, j, k+1} T_{i, j, k-1}=T_{i, j+1, k} T_{i, j-1, k}+\lambda_{i} T_{i+1, j, k} T_{i-1, j, k}
$$

As a consequence of the cluster algebra Laurent property [9], the solution $T_{i, j, k}$ of this equation may be expressed as a Laurent polynomial of any choice of admissible initial values, with coefficients in $\mathbb{Z}\left[\left(\lambda_{i}\right)_{i \in \mathbb{Z}}\right]$. The "coefficients" $\lambda_{i}$ are inhomogeneous, as they now depend on the value of $i$. In particular, choosing homogeneous coefficients $\lambda_{i}=\lambda$ independent of $i$, we recover the Laurent property observed for the Lambda-determinant of Robbins and Rumsey.

As it turns out, we may consider an even more general inhomogeneous coefficient $T$-system equation:

$$
T_{i, j, k+1} T_{i, j, k-1}=\mu_{j} T_{i, j+1, k} T_{i, j-1, k}+\lambda_{i} T_{i+1, j, k} T_{i-1, j, k}
$$

for any fixed non-vanishing coefficients $\left(\lambda_{i}\right)_{i \in \mathbb{Z}}$ and $\left(\mu_{j}\right)_{j \in \mathbb{Z}}$. We have the following

Theorem 8. The equation (3.2) is a particular mutation in a cluster algebra $\mathcal{A}$ with coefficents. The initial seed is given by the cluster $X_{0}=\left(\left(T_{i, j, 0}\right)_{i, j \in \mathbb{Z}},\left(T_{i^{\prime}, j^{\prime}, 1}\right)_{i^{\prime}, j^{\prime} \in \mathbb{Z}}\right)$ with $i+$ $j=1 \bmod 2$ and $i^{\prime}+j^{\prime}=0 \bmod 2$ as usual, and the coefficients $\left(\left(\lambda_{a}\right)_{a \in \mathbb{Z}},\left(\mu_{b}\right)_{b \in \mathbb{Z}}\right)$, and the extended exchange matrix $\tilde{B}_{0}$ with the following infinite block form: $\tilde{B}_{0}=\left(\begin{array}{cc}0 & B \\ -B & 0 \\ L_{0} & L_{1} \\ M_{0} & M_{1}\end{array}\right)$, 
where:

$$
\begin{array}{rlrl}
(B)_{i, j ; i^{\prime}, j^{\prime}} & =\delta_{i, i^{\prime}} \delta_{\left|j-j^{\prime}\right|, 1}-\delta_{j, j^{\prime}} \delta_{\left|i-i^{\prime}\right|, 1} \\
\left(L_{0}\right)_{a ; i, j} & =\delta_{i, a} & \left(L_{1}\right)_{a ; i^{\prime}, j^{\prime}}=-\delta_{i^{\prime}, a} \\
\left(M_{0}\right)_{b ; i, j} & =-\delta_{j, b} & \left(M_{1}\right)_{b ; i^{\prime}, j^{\prime}}=\delta_{j^{\prime}, b}
\end{array}
$$

Proof. We must show that the cluster $X_{k}=\left(\left(T_{i, j, k}\right)_{i, j \in \mathbb{Z}},\left(T_{i^{\prime}, j^{\prime}, k+1}\right)_{i^{\prime}, j^{\prime} \in \mathbb{Z}}\right)$, where $T_{i, j, k}$ is the solution of $(3.2)$ with prescribed values of $\left(\left(T_{i, j, 0}\right)_{i, j \in \mathbb{Z}},\left(T_{i^{\prime}, j^{\prime}, 1}\right)_{i^{\prime}, j^{\prime} \in \mathbb{Z}}\right)$, is a cluster in the cluster algebra $\mathcal{A}$. First let us represent the quiver $\tilde{Q}_{0}$ coded by the extended exchange matrix $\tilde{B}_{0}$. We represent by a filled $\bullet$ (resp. empty o) circle the vertices $(i, j)$ such that $i+j=1 \bmod 2\left(\operatorname{resp} .\left(i^{\prime}, j^{\prime}\right)\right.$ such that $\left.i^{\prime}+j^{\prime}=0 \bmod 2\right)$, and by a cross $\otimes($ resp. square $\square$ ) the vertices $(a)$ indexing $\lambda_{a}$ (resp. (b) indexing $\mu_{b}$ ) of $\tilde{Q}_{0}$. We have the following local structure, around respectively vertices $(i, j) \bullet$ and $\left(i^{\prime}, j^{\prime}\right) \circ$ :

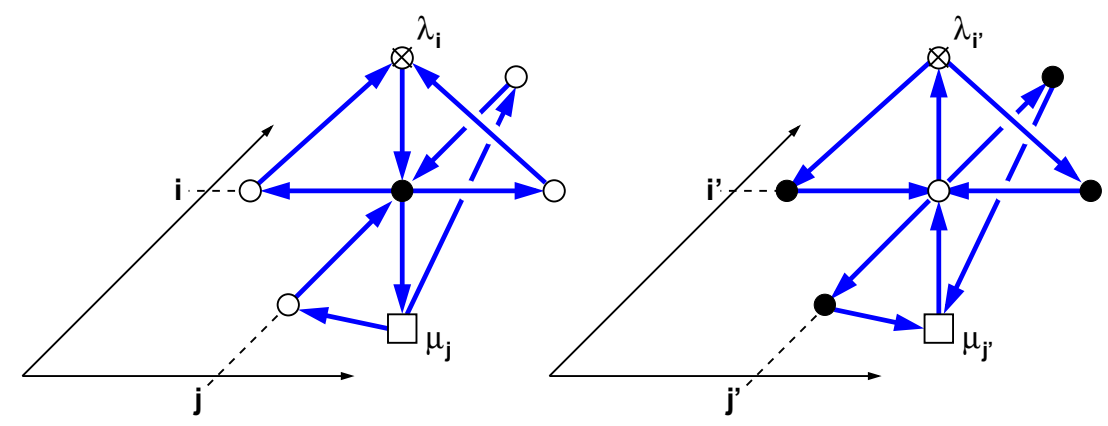

Note that the vertices $\left(a=i, i^{\prime}\right)$ indexing $\lambda_{i}, \lambda_{i^{\prime}}$ are connected to all $(i, j),\left(i^{\prime}, j\right)$ respectively, for all $j \in \mathbb{Z}$, while vertices $\left(b=j, j^{\prime}\right)$ indexing $\mu_{j}, \mu_{j^{\prime}}$ are connected to all $(i, j),\left(i, j^{\prime}\right)$ respectively, for all $i \in \mathbb{Z}$, with edges of alternating orientations. We may reach the cluster $X_{1}$ from the initial cluster $X_{0}$ by a compound mutation, obtained by mutating all the vertices $\bullet$. The resulting quiver $\tilde{Q}_{1}$ is identical to $\tilde{Q}_{0}$ with all edge orientations flipped, namely $\tilde{B}_{1}=-\tilde{B}_{0}$, as illustrated below for mutations in a quadrant around a $\bullet$ vertex:

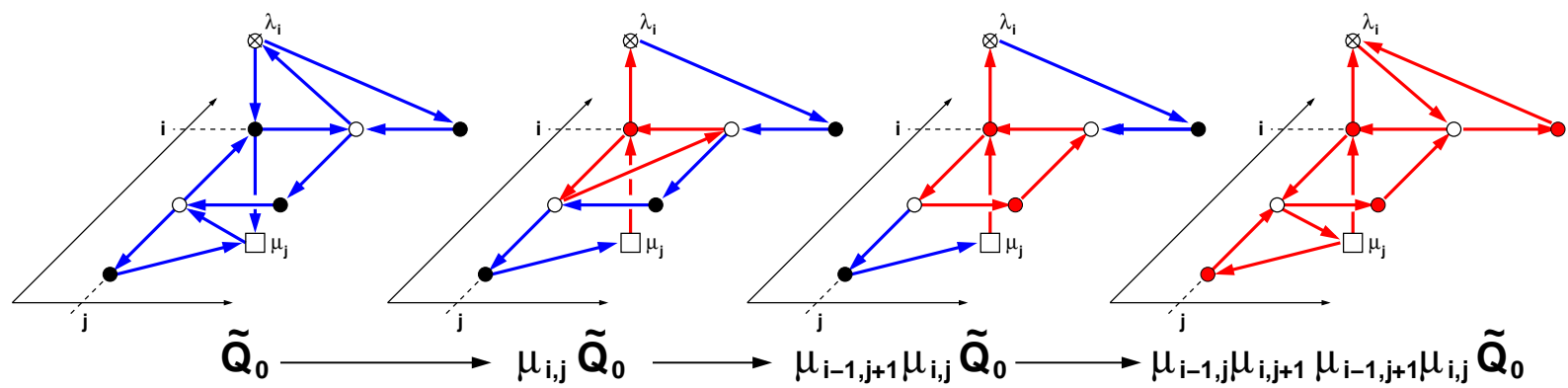

(Recall that a mutation at a vertex $v$ of a quiver flips the orientations of all incident edges, creates "shortcut" edges $u \rightarrow w$ for any length 2 path $u \rightarrow v \rightarrow w$ before mutation, and any 2-loop $v \rightarrow w \rightarrow v$ thus created must be eliminated.)

The cluster $X_{2}$ is then reached by mutating all vertices $\circ$, and has the exchange matrix $\tilde{B}_{2}=\tilde{B}_{0}$, etc. 
As a consequence of the theorem, we have the Laurent property: the solution $T_{i, j, k}$ of the system (3.2) for prescribed values of $\left(\left(T_{i, j, 0}\right)_{i, j \in \mathbb{Z}},\left(T_{i^{\prime}, j^{\prime}, 1}\right)_{i^{\prime}, j^{\prime} \in \mathbb{Z}}\right)$, is a Laurent polynomial of these values, with coefficients in $\mathbb{Z}\left[\left(\lambda_{a}\right)_{a \in \mathbb{Z}},\left(\mu_{b}\right)_{b \in \mathbb{Z}}\right]$.

\subsection{Generalized Lambda-determinant: definition and proper- ties}

In view of the results of previous section, we may recast the Definition 1 into the following:

Lemma 9. Given a square matrix $A=\left(a_{i, j}\right)_{i, j \in I}$, and some parameters $\lambda_{a}, \mu_{a}, a \in J=$ $\{-(n-2),-(n-3), \ldots,-1,0,1, \ldots, n-3, n-2\}$, the inhomogeneous Lambda-determinant of $A$ is the solution $T_{0,0, n}$ of the inhomogeneous $T$-system (3.2), with initial conditions $(2.3)$.

As a consequence of Theorem 8 , we know that $|A|_{\lambda ; \mu}$ is a Laurent polynomial of the entries $a_{i, j}$ of $A$, with coefficients in $\mathbb{Z}\left[\left(\lambda_{a}\right)_{a \in J},\left(\mu_{a}\right)_{a \in J}\right]$.

Example 10. The generalized Lambda-determinant of a $3 \times 3$ matrix A reads:

$$
\begin{aligned}
\left|\begin{array}{lll}
a & b & c \\
d & e & f \\
g & h & k
\end{array}\right|_{\lambda, \mu}= & \lambda_{1} \lambda_{0} \lambda_{-1} c e g+\lambda_{0} \mu_{0} \lambda_{1} c d h+\lambda_{0} \mu_{0} \lambda_{-1} b f g \\
& +\mu_{1} \mu_{0} \mu_{-1} a e k+\lambda_{0} \mu_{0} \mu_{1} b d k+\lambda_{0} \mu_{0} \mu_{-1} a f h+\lambda_{0} \mu_{0}\left(\lambda_{0}+\mu_{0}\right) \frac{b d f h}{e}
\end{aligned}
$$

Example 11. Let $\lambda_{a}=\lambda$ and $\mu_{a}=\mu$, for all $a$, and $A$ be the Vandermonde matrix $A=\left(a_{i}^{j-1}\right)_{i, j \in I}$. Then we have:

$$
|A|_{\lambda ; \mu}=\prod_{1 \leqslant i<j \leqslant n}\left(\lambda a_{i}+\mu a_{j}\right)
$$

The generalized Lambda-determinant reduces to the original Robbins-Rumsey Lambda-determinant for the choice of parameters $\lambda_{a}=\lambda$ and $\mu_{a}=1$ for all $a$.

The generalized Lambda-determinant satisfies a number of properties, inherited from the symmetries of the $T$-system. We have the following

Proposition 12. Let $\sigma, \tau$ be the following transformations of the matrix $A=\left(a_{i, j}\right)_{i, j \in I}$, corresponding respectively to quarter-turn rotation, and diagonal reflection:

$$
\sigma(A)_{i, j}=a_{n+1-j, i} \quad \tau(A)_{i, j}=a_{j, i}
$$

Then with the following action on parameters:

$$
\left\{\begin{array} { c } 
{ \sigma ( \lambda ) _ { a } = \mu _ { - a } } \\
{ \sigma ( \mu ) _ { a } = \lambda _ { a } }
\end{array} \quad \text { and } \quad \left\{\begin{array}{c}
\tau(\lambda)_{a}=\lambda_{-a} \\
\tau(\mu)_{a}=\mu_{a}
\end{array}\right.\right.
$$

we have: $|\varphi(A)|_{\varphi(\lambda), \varphi(\mu)}=|A|_{\lambda, \mu}$ for $\varphi=\sigma, \tau$. 
Proof. To compute $|\varphi(A)|_{\varphi(\lambda), \varphi(\mu)}$, we use the following $T$-system relation

$$
T_{i, j, k+1}^{\varphi} T_{i, j, k-1}^{\varphi}=\mu_{j}^{\varphi} T_{i, j+1, k}^{\varphi} T_{i, j-1, k}^{\varphi}+\lambda_{i}^{\varphi} T_{i+1, j, k}^{\varphi} T_{i-1, j, k}^{\varphi}
$$

with $\lambda_{i}^{\varphi}=\varphi(\lambda)_{i}$ and $\mu_{j}^{\varphi}=\varphi(\mu)_{j}$, together with the initial data

$$
T_{i, j, 0}^{\varphi}=1 \quad(i+j=n \bmod 2) \quad T_{i, j, 1}^{\varphi}=\varphi(A) \frac{j-i+n+1}{2}, \frac{i+j+n+1}{2} \quad(i+j=n+1 \bmod 2)
$$

We wish to compare the solution $T_{0,0, n}^{\varphi}$ with that, $T_{0,0, n}$, of the $T$-system (3.2) subject to the initial conditions (2.3). For $\varphi=\sigma, \tau$, we have respectively:

$$
\begin{aligned}
& T_{i, j, 1}^{\sigma}=\sigma(A)_{\frac{j-i+n+1}{2}, \frac{i+j+n+1}{2}}=a_{\frac{-i-j+n+1}{2}, \frac{-i+j+n+1}{2}}=T_{j,-i, 1} \\
& T_{i, j, 1}^{\tau}=\tau(A) \frac{j-i+n+1}{2}, \frac{i+j+n+1}{2}=a_{\frac{i+j+n+1}{2}, \frac{-i+j+n+1}{2}}=T_{-i, j, 1}
\end{aligned}
$$

From the obvious symmetries of the $T$-system, and with the respective choice of parameters (3.3), we deduce that

$$
T_{i, j, k}^{\sigma}=T_{j,-i, k} \quad \text { and } \quad T_{i, j, k}^{\tau}=T_{-i, j, k}
$$

for all $i, j, k$ such that $i+j+k=n \bmod 2$. The proposition follows for $i=j=0$ and $k=n$.

\subsection{A non-homogeneous example}

In this section we study a non-trivial example of an inhomogeneous generalized Lambdadeterminant that may be of interest as a statistical model. We choose the matrix $A=$ $\left(a_{i, j}\right)_{i, j \in I}$ with entries

$$
a_{i, j}=1 \quad(i, j \in I)
$$

We pick coefficients with an explicit dependence on their index, namely

$$
\lambda_{a}=q^{a} \quad \mu_{b}=q^{b} \quad(a, b \in \mathbb{Z})
$$

for some fixed parameter $q \in \mathbb{C}^{*}$. We have the following

Theorem 13. The generalized Lambda-determinant of the matrix $A$ (3.4) and with the parameters $\lambda, \mu$ of (3.5) reads:

$$
|A|_{\lambda, \mu}=\prod_{m=1}^{\left\lfloor\frac{n}{2}\right\rfloor} \prod_{j=2 m-n}^{n-2 m}\left(1+q^{j}\right)
$$

To prove the theorem, let us actually solve the more general $T$-system (3.2) with coefficients $\lambda_{a}, \mu_{b}$ as in (3.5), and with initial conditions (2.3). We have: 
Lemma 14. The solution of the inhomogeneous T-system (3.2) with coefficients $\lambda_{a}, \mu_{b}$ as in (3.5) and with initial data $T_{i, j, 0}=T_{i, j, 1}=1$ for all $i, j \in \mathbb{Z}$ reads:

$$
\begin{aligned}
T_{i, j, k}=q^{\frac{k(k-1)}{2} \operatorname{Min}(i, j)} & \prod_{m=1}^{\left\lfloor\frac{k-|i-j|}{2}\right\rfloor} \prod_{a=2 m-k+|i-j|}^{k-|i-j|-2 m}\left(1+q^{a}\right) \\
& \times \prod_{m=1}^{|i-j|} \prod_{a=m}^{k-|i-j|+2 m-2}\left(1+q^{a}\right)
\end{aligned}
$$

Proof. By uniqueness of the solution for the given initial data, we simply have to check that the above satisfies both the initial condition $T_{i, j, 0}=T_{i, j, 1}=1$ and the $T$-system with coefficients. The condition $T_{i, j, 0}=T_{i, j, 1}=1$ is clear from the formula (3.6). The expression (3.6) is easily shown to satisfy:

$$
\frac{T_{i, j+1, k} T_{i, j-1, k}}{T_{i+1, j, k} T_{i-1, j, k}}=1 \quad\left(i, j \in \mathbb{Z} ; k \in \mathbb{Z}_{+}\right)
$$

as the various factors cancel out exactly. Analogously, we find that only one factor survives in:

$$
\frac{T_{i, j, k+1} T_{i, j, k-1}}{T_{i+1, j, k} T_{i-1, j, k}}=q^{i}+q^{j} \quad\left(i, j \in \mathbb{Z} ; k \in \mathbb{Z}_{>0}\right)
$$

and therefore (3.2) follows, the lemma is proved.

Theorem 13 follows by taking $i=j=0, k=n$ in the expression (3.6) above. Note that the result of Theorem 13 reduces in the homogeneous limit $q \rightarrow 1$ to:

$$
T_{0,0, n}=2^{\frac{n(n-1)}{2}}
$$

in agreement with the result of Example 11 for $a_{i}=1$ for all $i$ and $\lambda=\mu=1$.

\section{Computing the generalized Lambda-determinant}

We first write a solution of the $T$-system with inhomogeneous coefficients, based on a matrix representation generalizing the solution of $[6]$.

\subsection{Inhomogeneous $T$-system solution I: $V$ and $U$ matrices, def- inition and properties}

Let us consider the square lattice with vertex set $\mathbb{Z}^{2}$, and its elementary triangulations, obtained by picking either of the two possible diagonal edges in each square face. We consider a pair of such triangles sharing an horizontal edge of the lattice as a generalized rhombus, and we restricted ourselves to bicolored triangulations such that exactly one of the two triangles in each generalized rhombus is grey, the other one being white. We 
moreover attach variables to the vertices of the lattice. This gives rise to eight possible generalized rhombi. In analogy with the solution of the $A_{r} T$-system [3], we associate the following $2 \times 2$ matrices to each of these generalized rhombi, with entries Laurent monomials of the variables at the three vertices adjacent to the grey triangle, and also depending on fixed coefficients $\lambda, \mu$ :

$$
\begin{aligned}
V(d, a, b ; \lambda, \mu) & =\left(\begin{array}{cc}
\mu \frac{a}{b} & \lambda \frac{d}{b} \\
0 & 1
\end{array}\right)={ }^{\prime}(d, a, b ; \lambda, \mu)=\left(\begin{array}{cc}
\frac{a}{b} & \lambda \frac{d}{b} \\
0 & 1
\end{array}\right)={ }^{\mathbf{b}} \text { or } \\
U(a, b, c ; \lambda, \mu) & =\left(\begin{array}{cc}
1 & 0 \\
\frac{c}{b} & \frac{a}{b}
\end{array}\right)=\mathbf{a} \\
U^{\prime}(a, b, c ; \lambda, \mu) & =\left(\begin{array}{cc}
1 & 0 \\
\frac{c}{b} & \mu \frac{a}{b}
\end{array}\right)=\mathbf{a}
\end{aligned}
$$

Note that we have represented a thicker blue horizontal (resp. red vertical) edge to indicate the presence of the parameter $\mu$ (resp. $\lambda$ ) in the corresponding matrices. Next, we introduce a graphical calculus, by associating to pictures some matrix products. We read pictures from left to right, and take the product of matrices in the same order.

With this rule, the above matrices satisfy the following property, easily checked by direct calculation.

\section{Lemma 15.}

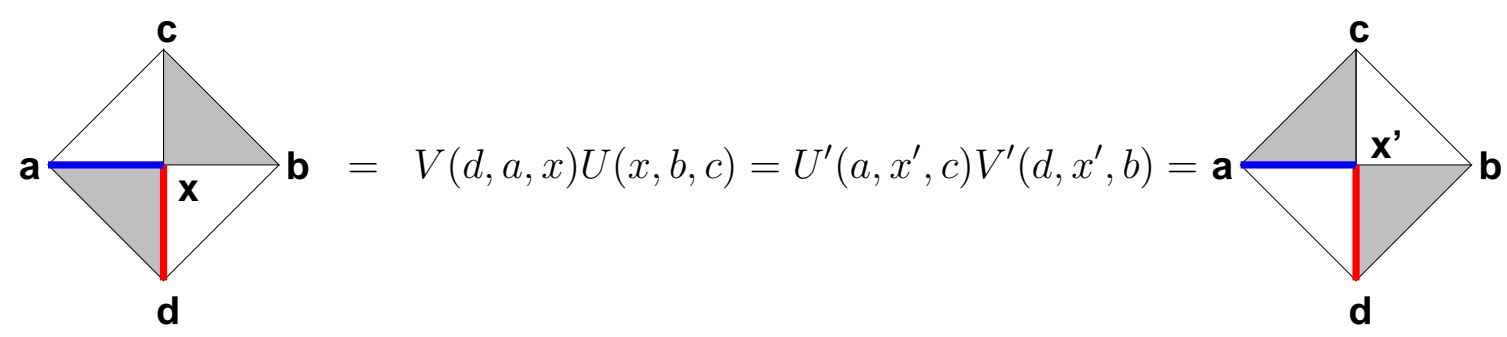

iff $\quad x x^{\prime}=\mu a b+\lambda c d$ 
We use the standard embeddings of any $2 \times 2$ matrix $M$ into $G L_{n}$ denoted $M_{i}, i=$ $1,2, \ldots, n-1$ with entries

$$
\left(M_{i}\right)_{r, s}=\left\{\begin{array}{cc}
\delta_{r, s} & \text { if } r \notin\{i, i+1\} \text { or } s \notin\{i, i+1\} \\
M_{r, s} & \text { otherwise }
\end{array}\right.
$$

In this embedding, it is clear that for any $2 \times 2$ matrices $M, P, M_{i}$ and $P_{j}$ commute for $|j-i|>1$. To avoid confusion, let us stress that the index $i$ of the $G L_{n}$ embedding must be understood as a vertical coordinate in the rhombus tiling picture (ranging from 1 to $n-1$, from bottom to top), which is strictly speaking "upside down" compared to the row index in the embedded matrices. We have also the following exchange properties, easily checked directly:

Lemma 16. The matrices $U_{i}, V_{j}$ satisfy:

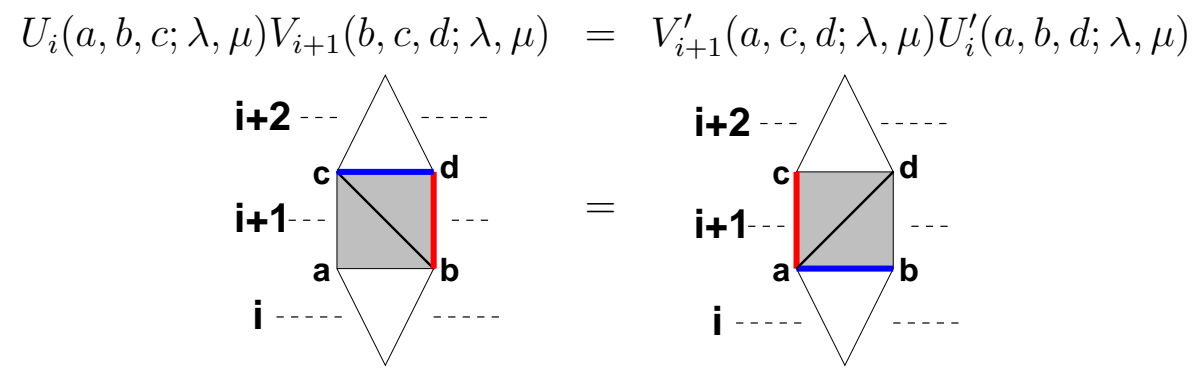

where again the pictures are read from left to right, the indices $i, i+1, \ldots$ from bottom to top, and the direction of the diagonal of a square indicates which generalized rhombus is to the left of the other, and we have represented the 4 up/down pointing white triangles in-between the two possible configurations of half-squares each of them may be in (total of 16 configurations here). We also have the commutation relations:

$$
\begin{aligned}
{\left[V_{i}(c, a, b), U_{j}(d, e, f)\right] } & =\left[V_{i}^{\prime}(c, a, b), U_{j}(d, e, f)\right]=\left[V_{i}(c, a, b), U_{j}^{\prime}(d, e, f)\right] \\
& =\left[V_{i}^{\prime}(c, a, b), U_{j}^{\prime}(d, e, f)\right]=0 \quad(j \neq i, i-1)
\end{aligned}
$$

The relations of Lemmas 15 and 16 are all we need to construct the solutions of the unrestricted $A_{\infty} T$-system with coefficients, expressed in terms of products of $U$ and $V$ matrices. The relation of Lemma 15 allows to implement the $T$-system evolution on the variable in the center $x \rightarrow x^{\prime}$. The relations of Lemma 16 allow for rearranging triangulations so as to be able to apply the former. In particular, when representing white and grey triangulations, we may omit the diagonal of each square of a uniform color, as both choices of diagonal lead to the same matrix product. So we will typically consider tilings of domains of $\mathbb{Z}^{2}$ by means of grey and white unit squares (2 tiles), and grey and white triangles equal to half a unit square, cut by one diagonal (4 tiles) such that any edge is common to two tiles of different colors. We call such a tiling a square-triangle tiling. 


\subsection{Inhomogeneous $T$-system solution II: a determinant formula}

We consider the tilted square domain $D_{k}(i, j) \subset \mathbb{Z}^{2}$ defined by

$$
D_{k}(j, i)=\left\{(x, y) \in \mathbb{Z}^{2} \text { such that }|x-j|+|y-i| \leqslant k-1\right\} \quad\left(i, j \in \mathbb{Z} ; k \in \mathbb{Z}_{+}\right)
$$

We also consider two square-triangle tilings of $D_{k}(j, i)$, denoted by $\theta_{\min }(k)$ and $\theta_{\max }(k)$ defined as follows. $\theta_{\min }(k)$ is the intersection of a checkerboard of alternating grey and white squares with $D_{k}(j, i)$ such that the NW border of $D_{k}(j, i)$ is adjacent only to white triangles (likewise, the SE border is adjacent to only white triangles, while the SW and NE borders are adjacent only to black triangles). $\theta_{\max }(k)$ is the only square-triangle tiling without squares having opposite color boundary assignments (NW,SE grey, SW,NE white). Here are examples of $\theta_{\min }(k)$ and $\theta_{\max }(k)$ for $k=5$ :
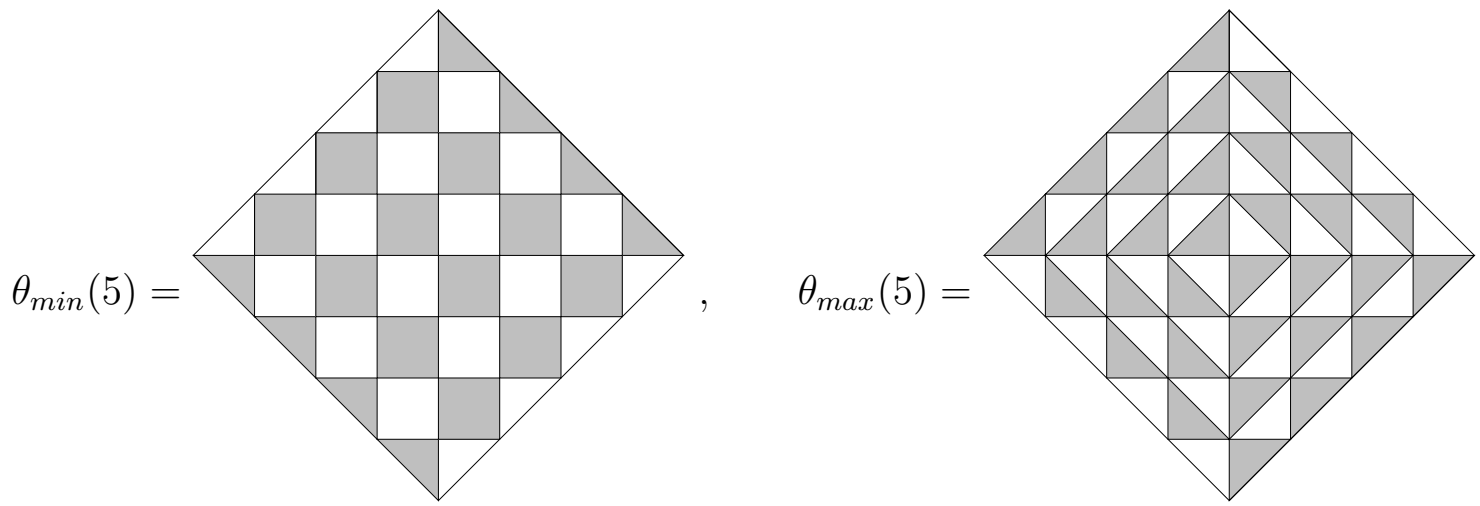

To attach matrices to these tilings, let us also include thick (red or blue) edges like in the representations of $U, V, U^{\prime}, V^{\prime}$. We fix the ambiguity on grey squares of $\theta_{\min }(k)$ (from Lemma 16) by picking the l.h.s. representation. This gives two decorated tilings $\tilde{\theta}_{\min }(k)$ and $\tilde{\theta}_{\max }(k)$ which for $k=5$ read:
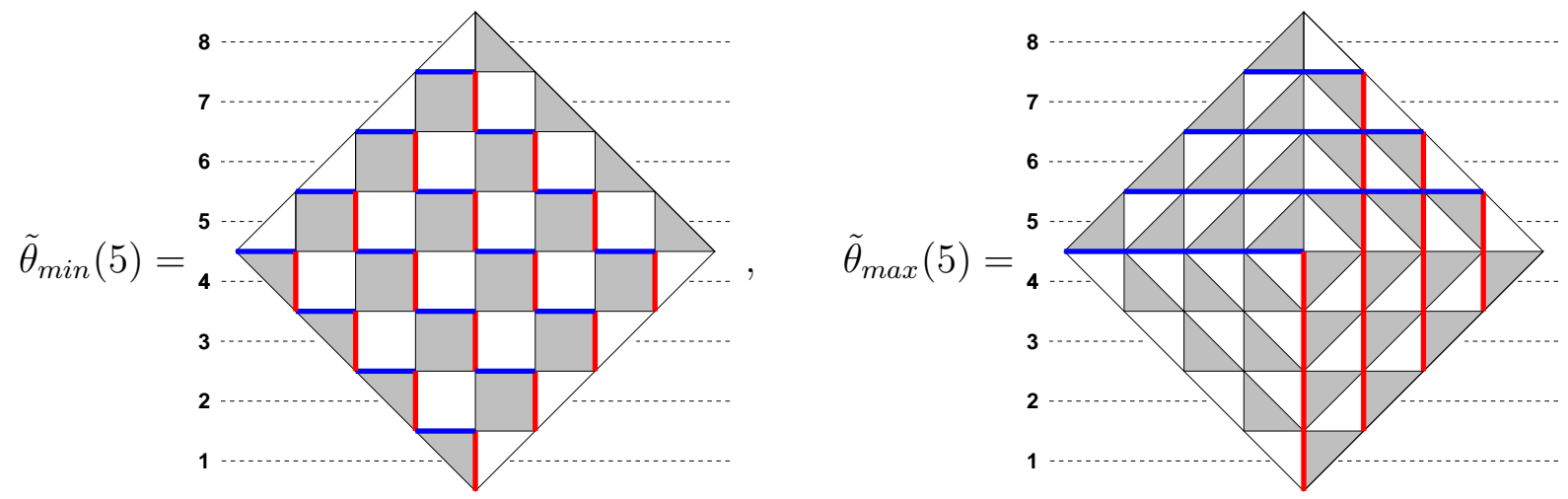

We finally attach variables to the vertices and coefficients to the thickened edges of the tilings as follows. On $\tilde{\theta}_{\min }(k)$ we assign variables $t_{i, j}$ to the vertices $(j, i) \in D_{k}(0,0)$, and coefficients $\lambda_{i}$ to thickened vertical (red) edges with top vertex of the form $(\ell, i)$, $\mu_{j}$ to thickened horizontal (blue) edges with right vertex of the form $(j, m), \ell, m \in \mathbb{Z}$. On $\tilde{\theta}_{\text {max }}(k)$ we assign variables $u_{i, j}$ to the vertices $(j, i) \in D_{k}(0,0)$, and coefficients $\lambda_{i}$ to 
thickened vertical (red) edges with top vertex of the form $(\ell, i), \mu_{j}$ to thickened horizontal (blue) edges with right vertex of the form $(j, m), \ell, m \in \mathbb{Z}$. For completeness, we assign the value $\mu=1$ (resp. $\lambda=1$ ) to coefficients corresponding to non-thickened horizontal (resp. vertical) edges.

We finally attach matrices $\Theta_{\min }(k)$ and $\Theta_{\max }(k)$ to the two tilings. These are defined as the products of $U_{i}, V_{i}, U_{i}^{\prime}, V_{i}^{\prime}$ matrices embedded into $G L_{2 k-2}$, coded respectively by the two decorated tilings with assigned vertex and thickened edge values. Note again that the index $i$ is to be read from bottom to top in the rhombus tiling, whereas it is read from top to bottom as usual in the $G L_{2 k-2}$ matrices. Likewise, the directions NW,SW,NE,SE refer to the domain $D_{k}(j, i)$ or its tiling configurations $\tilde{\theta}_{\min , \max }(k)$, not the corresponding matrices $\Theta_{\min , \max }(k)$, which are upside-down in comparison.

Example 17. For $k=3$, we have the $4 \times 4$ matrix:

$$
\begin{aligned}
\Theta_{\min }(3)= & V_{2}\left(t_{-1,-1}, t_{0,-2}, t_{0,-1} ; \lambda_{0}, \mu_{-1}\right) V_{1}\left(t_{-2,0}, t_{-1,-1}, t_{-1,0} ; \lambda_{-1}, \mu_{0}\right) \\
& \times U_{2}\left(t_{0,-1}, t_{0,0}, t_{1,-1} ; 1,1\right) V_{3}\left(t_{0,0}, t_{1,-1}, t_{1,0} ; \lambda_{1}, \mu_{0}\right) \\
& \times U_{1}\left(t_{-1,0}, t_{-1,1}, t_{0,0} ; 1,1\right) V_{2}\left(t_{-1,1}, t_{0,0}, t_{0,1} ; \lambda_{0}, \mu_{1}\right) \\
& \times U_{3}\left(t_{1,0}, t_{1,1}, t_{2,0} ; 1,1\right) U_{2}\left(t_{0,1}, t_{0,2}, t_{1,1} ; 1,1\right)
\end{aligned}
$$

corresponding to the following variables and coefficients:

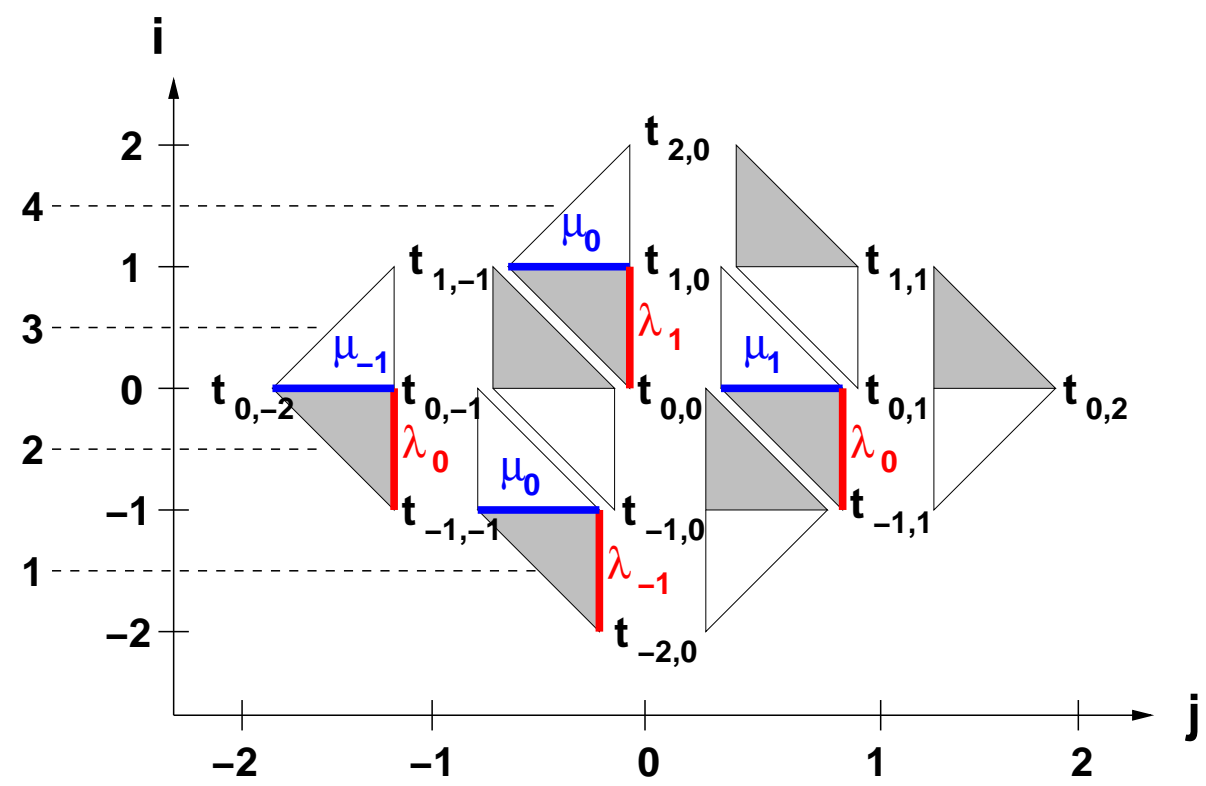

Likewise, we have:

$$
\begin{aligned}
\Theta_{\max }(3)= & U_{2}^{\prime}\left(u_{0,-2}, u_{0,-1}, u_{1,-1} ; 1, \mu_{-1}\right) U_{1}\left(u_{-1,-1}, u_{-1,0}, u_{0,-1} ; 1,1\right) \\
& \times U_{3}^{\prime}\left(u_{1,-1}, u_{1,0}, u_{2,0} ; 1, \mu_{0}\right) U_{2}^{\prime}\left(u_{0,-1}, u_{0,0}, u_{1,0} ; 1, \mu_{0}\right) \\
& \times V_{2}^{\prime}\left(u_{-1,0}, u_{0,0}, u_{0,1} ; \lambda_{0}, 1\right) V_{1}^{\prime}\left(u_{-2,0}, u_{-1,0}, u_{-1,1} ; \lambda_{-1}, 1\right) \\
& \times V_{3}\left(u_{0,1} u_{1,0}, u_{1,1} ; \lambda_{1}, \mu_{1}\right) V_{2}^{\prime}\left(u_{-1,1}, u_{0,1}, u_{0,2} ; \lambda_{0}, 1\right)
\end{aligned}
$$


corresponding to the following variables and coefficients:

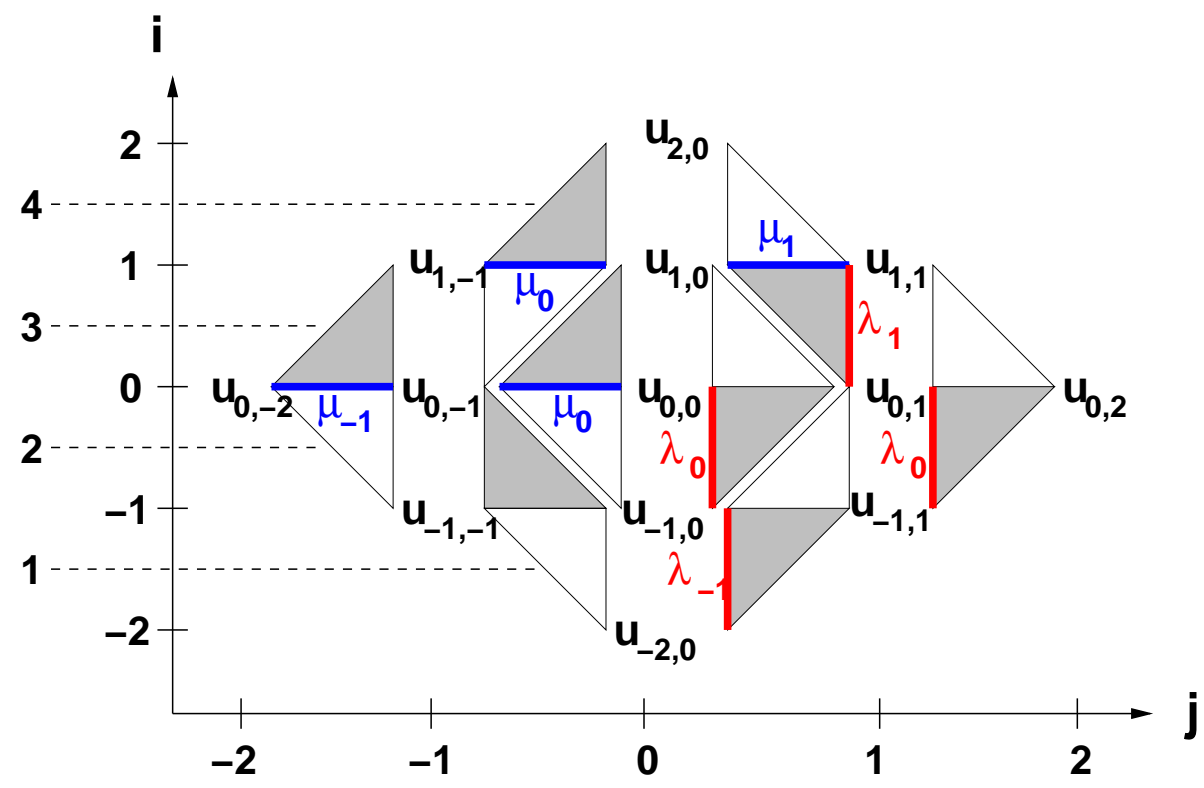

We have:

Theorem 18. Let $n \geqslant 1$, and let us pick variables $u_{i, j}$ as:

$$
u_{i, j}=T_{i, j, n-|i|-|j|} \quad(j, i) \in D_{n}(0,0)
$$

where $T_{i, j, k}$, for $i+j+k=n$ mod 2, is the solution of the $T$-system with coefficients (3.2) subject to the initial conditions

$$
T_{i, j, \epsilon_{i, j, n}}=t_{i, j} \quad(j, i) \in D_{n}(0,0)
$$

where $\epsilon_{i, j, n}=(i+j+n \bmod 2) \in\{0,1\}$. Then we have the matrix identity

$$
\Theta_{\min }(n)=\Theta_{\max }(n)
$$

Proof. The identity is proved by showing that we may transform the minimal tiling $\tilde{\theta}_{\min }(n)$ with variables $t_{i, j}$ into the maximal tiling $\theta_{\max }(n)$ with variables $u_{i, j}$ by successive applications of the Lemmas 15 and 16. This is illustrated below for the case $n=3$.

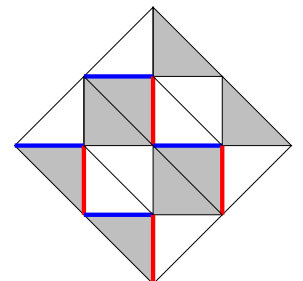

(a)

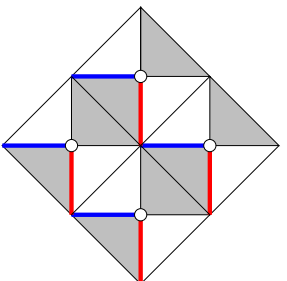

(b)

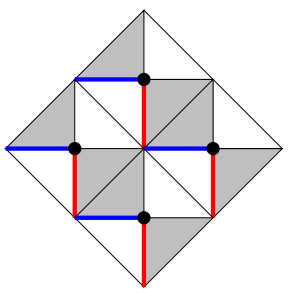

(c)

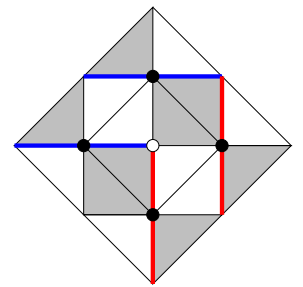

(d)

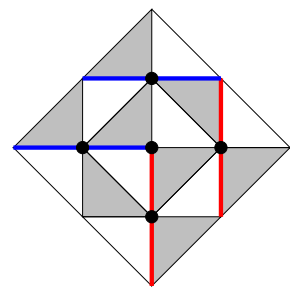

(e)

Starting from $\tilde{\theta}_{\text {min }}(n)$ (a), let us first apply Lemma 16 to flip the diagonals of all the white squares (b). Then apply Lemma 15 to update all the values at vertices $(j, i) \in D_{n}(0,0)$ 
such that $i+j=n \bmod 2$ (marked as empty circles on (b)). Due to the update relation $x x^{\prime}=\mu a b+\lambda c d$, and comparing with the $T$-system evolution (3.2), we see that the updated values are $T_{i, j, 2}$ (filled vertices on $(\mathrm{c})$ ). We then use Lemma 16 to flip the diagonals of all the remaining squares $(\mathrm{d})$, then update the values at vertices $(j, i) \in D_{n-1}(0,0)$ such that $i+j=n-1 \bmod 2$ (empty circle on $(\mathrm{d})$ ), yielding updated values $T_{i, j, 3}$. The procedure is iterated until there are no more squares. In the final tiling configuration, the boundary triangles have opposite colors from those of $\tilde{\theta}_{\min }(n)$, the tiling is therefore $\tilde{\theta}_{\max }(n)$. Note that the last (central) updated value is $u_{0,0}=T_{0,0, n}$. As both Lemmas 15 and 16 leave the result of the corresponding matrix products unchanged, the theorem follows.

Corollary 19. Let $a_{\min }(n), a_{\max }(n)$ denote respectively the $n-1 \times n-1$ initial principal minors of $\Theta_{\min }(n)$ and $\Theta_{\max }(n)$, then we have:

$$
a_{\min }(n)=a_{\max }(n)
$$

Our last task is to relate the central value $T_{0,0, n}=u_{0,0}$ to the matrix $\Theta_{\min }(n)$. We have the following:

Theorem 20. Notations are as in Theorem 18. The solution $T_{0,0, n}$ of the $T$ system with coefficients (3.2) is expressed in terms of its initial data $t_{i, j}$ as:

$$
T_{0,0, n}=a_{m i n}(n) \prod_{i=2-n}^{-1} t_{i, 1-n-i}^{-1} \prod_{i=2-n}^{0} t_{i, n-1+i}
$$

Proof. Let us compute $a_{\text {max }}(n)$. We note that, as $U_{i}, U_{i}^{\prime}$ are lower triangular and $V_{i}, V_{i}^{\prime}$ are upper triangular, the $2 n-2 \times 2 n-2$ matrix $\Theta_{\max }(n)$ is the product of a lower triangular $\mathcal{L}$ matrix by an upper one $\mathcal{U}$, corresponding respectively to the left and right halves of $\tilde{\theta}_{\text {max }}(n)$. More precisely, all matrices corresponding to the SW corner of the tiling $\tilde{\theta}_{\text {max }}(n)$ are of $U_{i}$ type $(i \leqslant n-2)$, all matrices in the NW corner of $\tilde{\theta}_{\text {max }}(n)$ are of $U_{i}^{\prime}$ type $(n-1 \leqslant i \leqslant 2 n-3)$, all matrices in the SE corner of $\tilde{\theta}_{\text {max }}(n)$ are of $V_{i}^{\prime}$ type $(i \leqslant n-1)$ and all matrices in the NE corner of $\tilde{\theta}_{\max }(n)$ are of $V_{i}$ type $(n \leqslant i \leqslant 2 n-3)$.

Let $M_{\max }(n)$ be the truncation of $\Theta_{\max }(n)$ to its $n-1$ first rows and columns. From the triangularity property, we have $M_{\max }(n)=\ell u$ where $\ell$ and $u$ are the truncations of $\mathcal{L}$ and $\mathcal{U}$ to their $n-1$ first rows and columns, so that $a_{\max }(n)=\operatorname{det}(\ell) \operatorname{det}(u)$. By the triangularity property, and the above remark on corners, $\operatorname{det}(\ell)$ is the product over all diagonal matrix elements of the $U_{i}$ in $\Theta_{\max }(n)$, while $\operatorname{det}(u)$ is the product over all diagonal matrix elements of the $V_{i}^{\prime}$ in $\Theta_{\max }(n)$. These in turn have a very simple interpretation in terms of the tilings: the diagonal matrix elements of $U_{i}(i \leqslant n-2)$ are the ratios (left vertex value)/(right vertex value) along the horizontal edges of the tiling (here in the strict SW corner, i.e. with $2-n \leqslant i<0$ and $1-n \leqslant j \leqslant 0$. The product over these is telescopic and leaves us with only the ratios of (leftmost vertex value)/(rightmost vertex value $)=u_{i, i+1-n} / u_{i, 0}$ along the row $i$. Analogously, the diagonal matrix elements of $V_{i}^{\prime}$ $(i \leqslant n-1)$ have the same interpretation, and we get the telescopic products $u_{i, 0} / u_{i, n-1+i}$ along the row $i$. Collecting both products, we get

$$
a_{\text {max }}(n)=\operatorname{det}(\ell) \operatorname{det}(u)=\prod_{i=1-n}^{-1} \frac{u_{i, 1-n-i}}{u_{i, 0}} \prod_{i=2-n}^{0} \frac{u_{i, 0}}{u_{i, n-1+i}}=u_{0,0} \prod_{i=2-n}^{-1} u_{i, 1-n-i} \prod_{i=2-n}^{0} u_{i, n-1+i}^{-1}
$$

THE ELECTRONiC JOURNAL OF COMBINATORICS 20(3) (2013), \#P19 
Finally, we note that the SW and SE boundary values of $u_{i, j}$ are all of the form $T_{i, j, 1}$ and therefore are equal to the corresponding $t_{i, j}$ variables. The theorem then follows from Corollary 19, allowing to substitute $a_{\min }(n)$ for $a_{\max }(n)$.

\subsection{A determinant formula for the generalized Lambda- determinant}

To get a formula for the generalized Lambda-determinant, we simply have to combine the results of Theorems 18 and 20. Our generalized Lambda-determinant is the solution $T_{0,0, n}$ of the $T$-system with coefficients subject to the initial conditions (2.3). It corresponds to the initial values:

$$
t_{i, j}=\left\{\begin{array}{cc}
1 & \text { if } i+j+n=0 \bmod 2 \\
a_{\frac{i-i+n+1}{2}, \frac{j+i+n+1}{2}} & \text { otherwise }
\end{array}\right.
$$

We assume from now on that the initial data $t_{i, j}$ is chosen as in (4.3). With the same notations as in the previous section, we obtain:

Theorem 21. The generalized Lambda-determinant of any matrix $A=\left(a_{i, j}\right)_{1 \leqslant i, j \leqslant n}$ reads:

$$
|A|_{\lambda, \mu}=a_{\min }(n) \prod_{i=2}^{n} a_{i, 1}^{-1} \prod_{i=1}^{n} a_{n, i}
$$

As such, it is a Laurent polynomial of the $a_{i, j}$ 's with coefficients in $\mathbb{Z}_{+}\left[\left(\lambda_{i}\right)_{i \in J},\left(\mu_{i}\right)_{i \in J}\right]$, where $J=\{1-n, 2-n, \ldots, n-1\}$.

Proof. By direct application of Theorems 18 and 20, with the initial conditions (4.3). We simply use the relation $t_{i, j}=a_{\frac{j-i+n+1}{2}, \frac{j+i+n+1}{2}}$ which gives $\prod_{i=2-n}^{-1} t_{i, 1-n-i}^{-1}=\prod_{i=2}^{n-1} a_{i, 1}^{-1}$ and $\prod_{i=2-n}^{0} t_{i, n-1+i}=\prod_{i=2}^{n} a_{n, i}$, and for convenience we insert extra terms $a_{n, 1}^{-1}\left(\right.$ resp. $\left.a_{n, 1}\right)$ in each product, that cancel out. As $a_{\min }(n)$ is an initial principal minor of a product of $U_{i}, U_{i}^{\prime}, V_{i}, V_{i}^{\prime}$ matrices, and as the entries of all the matrices are Laurent monomials of the $t_{i, j}$ 's and monomials of the $\lambda_{i}$ 's and $\mu_{j}$ 's, we recover the Laurent polynomiality property of cluster algebras with coefficients, namely that the solution $T_{0,0, n}$ is a Laurent polynomial of the entries of $A$, with coefficients that are polynomials of the $\lambda_{i}$ 's and $\mu_{j}$ 's. Moreover, from the form of the matrices, these polynomial coefficients have themselves non-negative integers coefficients. The theorem follows.

\section{From networks to $6 \mathrm{~V}$ model}

\subsection{Network formulation}

The matrices $V, V^{\prime}, U, U^{\prime}$ may be interpreted as elementary "chips" that may be used to build networks, i.e. directed graphs with weighted edges. Each non-zero matrix element 
$m_{i, j}$ of $V, V^{\prime}, U, U^{\prime}$ is interpreted as the weight of a directed edge connecting an entry vertex $i$ to an exit vertex $j$. This gives rise to the four following "chips":

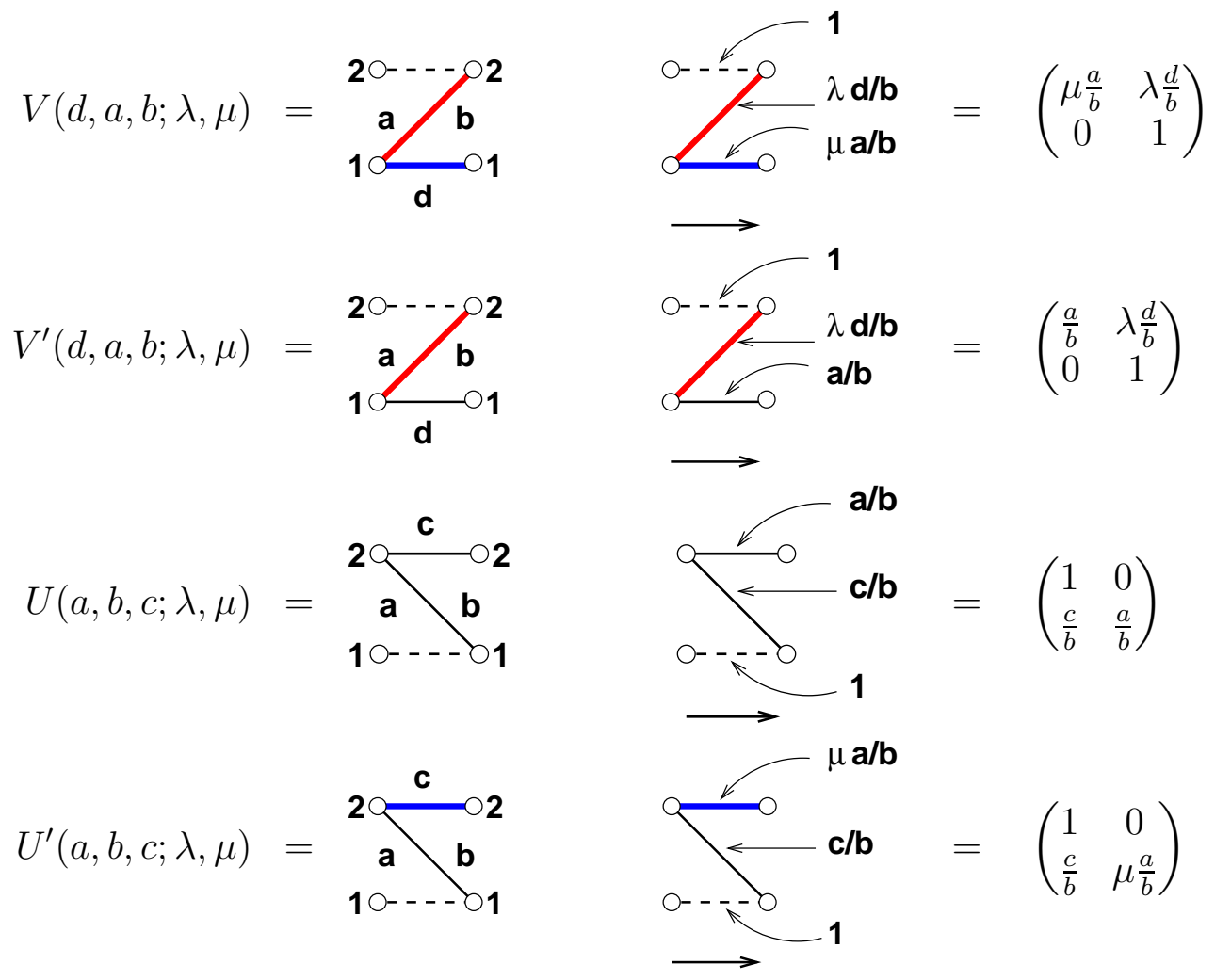

where we have represented in dashed black lines the edges with weight 1 , and in thickened red (resp. blue) edges the diagonals (resp. horizontals) carrying an extra multiplicative weight $\lambda$ (resp. $\mu$ ). The $G L_{r}$ embedding $M_{i}$ is clear: we place the chip for $M$ in positions $i, i+1$ and complete the graph by horizontal edges $j \rightarrow j$ with weight 1 , for $j \neq i, i+1$. Note that as for rhombus tilings, the index $i$ in networks is read from bottom to top, i.e. upside-down compared to the actual matrices. The product of two matrices correspond in this language to the concatenation of the graphs, namely the identification of the exit points of the left matrix graph with the entry points of the right matrix graph. Graphs obtained by concatenation of elementary chips are called networks. Note that in the present case networks have face labels, that determine all the edge weights via the rules (5.1).

The matrix of the network is the product of the matrices of the chips forming it. The matrix element $(i, j)$ of this product is the sum over all directed paths on the network with entry $i$ and exit $j$ of the product of the weights of the traversed edges.

We may now represent the networks $G_{\min }(n)$ and $G_{\max }(n)$ corresponding to the matrices $\Theta_{\min }(n)$ and $\Theta_{\max }(n)$. They are constructed as follows. Each lozenge in the decomposition of the corresponding tilings $\tilde{\theta}_{\min , \max }(n)$ is replaced by the corresponding chip from (5.1), at the same position. More precisely, a lozenge for the matrix $U_{i}, U_{i}^{\prime}, V_{i}, V_{i}^{\prime}$ is replaced by the corresponding embedded network chip with entry and exit points $i, i+1$. 
For $n=5$, this gives (recall that dashed black lines have weight 1 , while thickened blue (resp. red) lines have an extra weight $\mu$ (resp. $\lambda)$ ):

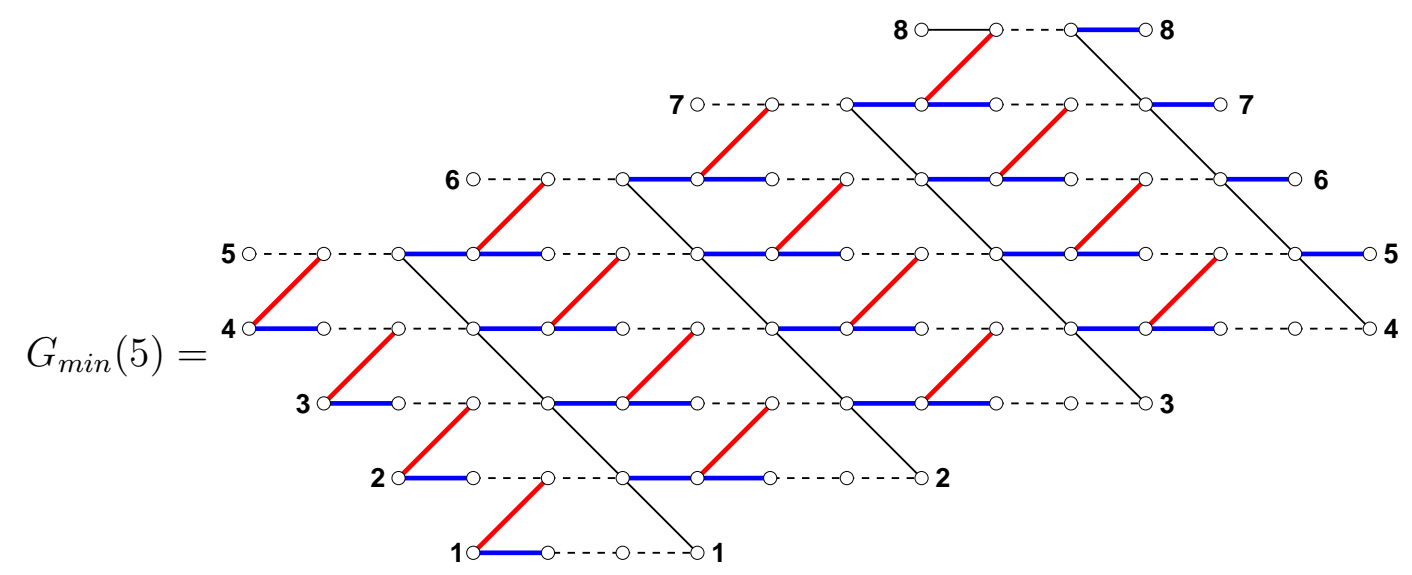

and

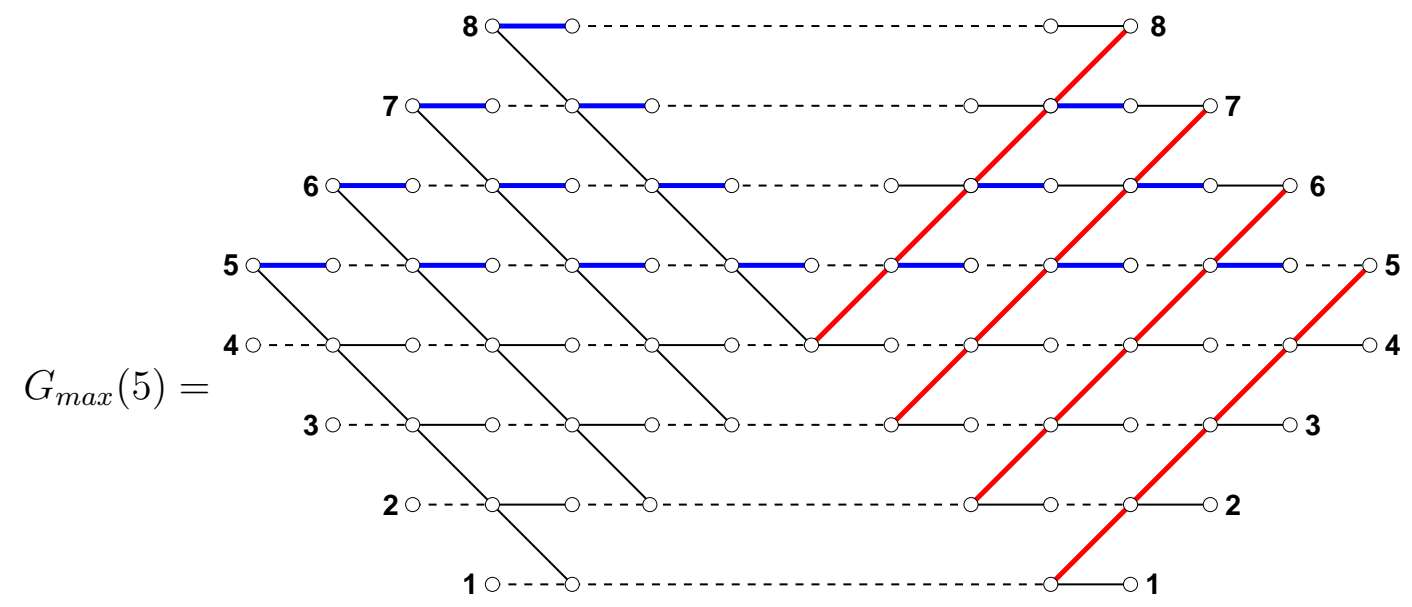

We have:

Lemma 22. The function $a_{\min }(n)$ (resp. $\left.a_{\max }(n)\right)$ is the partition function for families of non-intersecting paths that start at the entry points $1,2, \ldots, n-1$ and end at the exit points $1,2, \ldots, n-1$ of the network $G_{\min }(n)$ (resp. $\left.G_{\max }(n)\right)$.

Proof. By direct application of the Lindström-Gessel-Viennot Theorem $[11,12]$, as $a_{\min }(n)$ (resp. $\left.a_{\max }(n)\right)$ are the $n-1 \times n-1$ initial principal minors of $\Theta_{\min }(n)\left(\operatorname{resp} . \Theta_{\max }(n)\right)$, the matrices of the networks $G_{\min }(n)\left(\operatorname{resp} . G_{\max }(n)\right)$.

Remark 23. Note that due to the structure of $G_{\max }(n)$, there is a unique configuration of $n-1$ non-intersecting paths contributing to $a_{\max }(n)$, namely that in which all the paths have only horizontal steps. This gives an alternative pictorial interpretation of the proof of Theorem 20.

In view of Theorem 21, let us now reformulate $a_{\min }(n)$. To this effect, we will simplify slightly the network $G_{\min }(n)$ by use of the following: 
Lemma 24. We have the following equivalence between networks corresponding to the matrix product $U_{i-1}(a, b, c ; 1,1) V_{i}(b, c, d ; \lambda, \mu)$ :
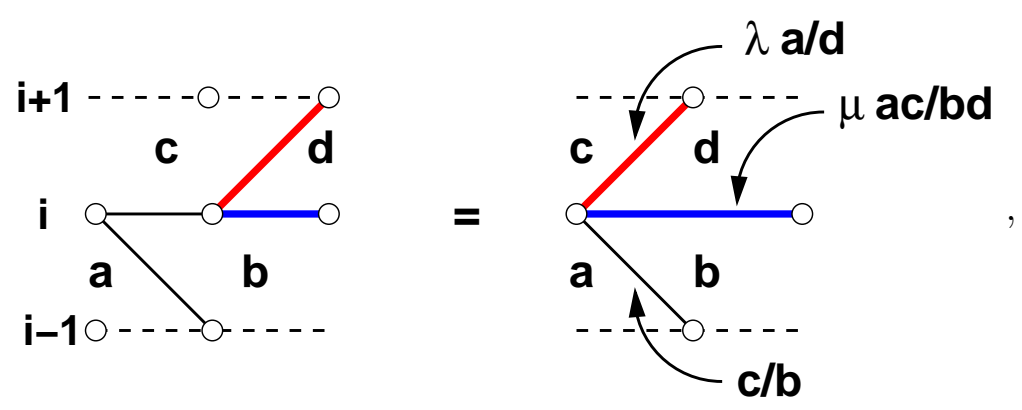

where we have indicated the new edge weights (and kept the color code for thickened edges receiving extra multiplicative weights $\mu$ (blue) and $\lambda$ (red).

Applying Lemma 24 to all the pieces corresponding to products $U_{i-1} V_{i}$ in the network $G_{\min }(n)$ allows to transform it into a new network $\tilde{G}_{\min }(n)$ with the same network matrix, but which is now a subset of the directed triangular lattice (with edges oriented from left to right). For $n=4$ this gives:

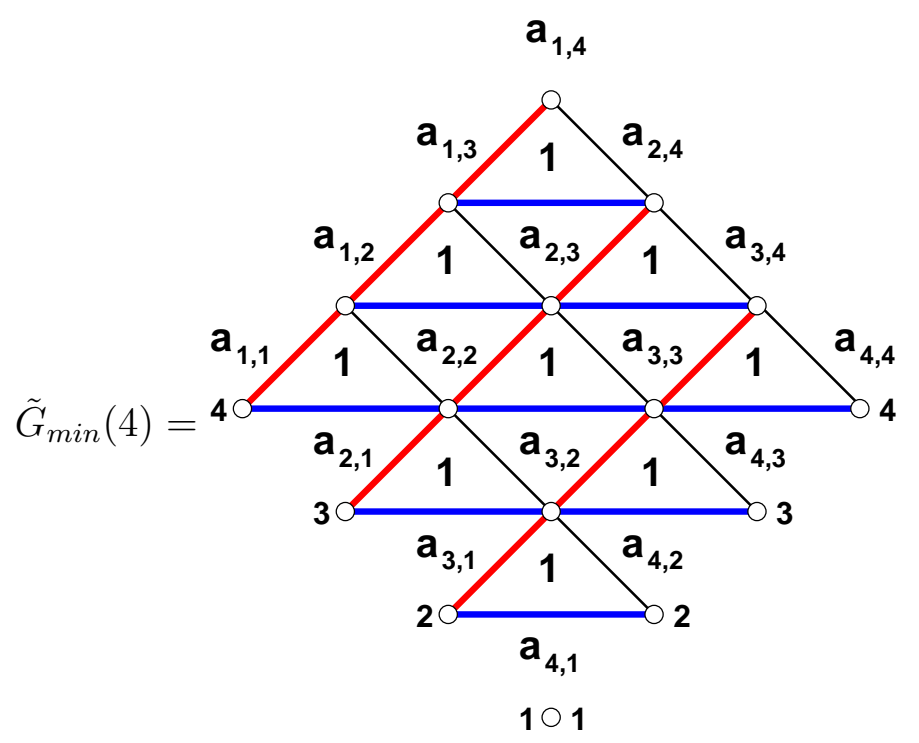

where we have indicated the face variables corresponding to the initial data (2.3). Note that the face labels of all up-pointing triangles are 1, whereas the down-pointing ones are the matrix elements of $A$. Note also that for technical reasons we have included a bottom isolated vertex. The matrix associated with this network, $\tilde{\Theta}_{\min }(n)$, has therefore size $2 n-1 \times 2 n-1$. The entry point 1 is trivially identified with the exit point 1 . 
The edge weights read as follows:

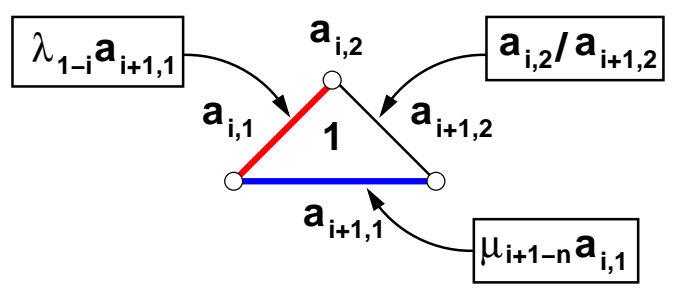

(a)

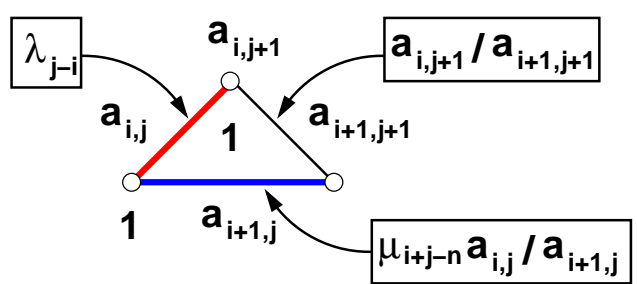

(b)

depending on whether the triangle with face label $a_{i, j}$ is on the SW border (a) for $j=1$ or anywhere else (b) for $j>1$. In view of the formula of Theorem 21, it is natural to absorb the prefactor $\prod_{i=2}^{n} a_{i, 1}^{-1}$ into a redefinition of the network $\tilde{G}_{m i n}$. Indeed, dividing the weights of the thickened colored edges in $(a)$ by $a_{i+1,1}$, for $i=1,2, \ldots, n-1$ exactly absorbs the prefactor, as the paths starting at the left vertex must either traverse the red or the blue adjacent edge. After this transformation, all the weights have the form (b) of (5.2) for $1 \leqslant i, j \leqslant n-1$, including the ones on the SW border. Denoting by $\hat{G}_{m i n}(n)$ the network thus modified, and by $\hat{a}_{m i n}(n)$ the $n \times n$ initial principal minor of the associated matrix $\hat{\Theta}_{\min }(n)$, we have the following reformulation of the generalized Lambda-determinant:

Theorem 25. The generalized Lambda-determinant of $A$ is given by:

$$
|A|_{\lambda, \mu}=\hat{a}_{\min }(n) \prod_{i=1}^{n} a_{n, i}
$$

As such, it is the partition function of families of n non-intersecting paths on the (directed triangular lattice) network $\hat{G}_{\text {min }}(n)$, starting from the $n S W$ vertices and ending at the $n$ $S E$ vertices, multiplied by all the entries of the last row of $A$.

\subsection{Another determinant formula for the generalized Lambda- determinant}

Let us now briefly describe a method for explicitly computing the generalized Lambdadeterminant of a given matrix $A$. The idea is to generate the paths eventually contributing to the minor $\hat{a}_{\min }(n)$. We consider points $(x, y)$ in the integer plane $\mathbb{Z}^{2}$, and a Hilbert space $H$ with a distinguished canonical basis $|x, y\rangle, x, y \in \mathbb{Z}$, and dual basis $\langle u, v|, u, v \in \mathbb{Z}$ such that $\langle u, v \mid x, y\rangle=\delta_{u, x} \delta_{v, y}$. Let us introduce operators $\hat{z}, \hat{w}$ acting on $H$ as follows:

$$
\hat{w}|x, y\rangle=|x+1, y+1\rangle \quad \hat{z}|x, y\rangle=|x+1, y-1\rangle
$$

Consider now the embedding of the network $\hat{G}_{\min }(n)$ into $\mathbb{Z}^{2}$, with vertices $(x, y) \in \mathcal{D}_{n}$, with:

$$
\mathcal{D}_{n}=\left\{(x, y) \in \mathbb{Z}^{2} \text { such that } x+y=0 \bmod 2 \text { and }|x|+|y-n+1| \leqslant n-1\right\}
$$


Note that the bottom vertex of $\hat{G}_{\min }(n)$ is now at the origin $(0,0)$. The starting points of the paths contributing to $\hat{a}_{\text {min }}(n)$ are $s_{i}=(1-i, i-1), i=1,2, \ldots, n$ and the endpoints are $e_{j}=(j-1, j-1), j=1,2, \ldots, n$. To each vertex $(x, y) \in \mathcal{D}_{n}$, we attach the label of the adjacent down-pointing triangle of $\hat{G}_{\min }(n),\{(x-1, y+1),(x+1, y+1),(x, y)\}$. For simplicity, we denote by $\alpha_{x, y}=a_{\frac{x-y}{2}+n, \frac{x+y}{2}+1}$ this label. We also attach the parameters $\ell_{y}=\lambda_{y+1-n}$ and $m_{x}=\mu_{x}$ respectively corresponding to the weights of the adjacent edges $(x, y)-(x+1, y+1)$ and $(x-1, y+1)-(x+1, y+1)$ of $\hat{G}_{m i n}(n)$. This leads to the natural definition of the following diagonal operators:

$$
\hat{\alpha}|x, y\rangle=\alpha_{x, y}|x, y\rangle, \quad \hat{\ell}|x, y\rangle=\ell_{y}|x, y\rangle, \quad \hat{m}|x, y\rangle=m_{x}|x, y\rangle
$$

Starting from a vertex $(x, y)$ there are three possibilities of steps in $\hat{G}_{\min }(n)$, with the weights described in (5.2) (b). These weighted steps are generated by the following operator acting on $H$ :

Definition 26. We define the transfer operator $T$, acting on $H$ as follows:

$$
\begin{aligned}
T & =\hat{w} \hat{\ell}+\hat{w} \hat{m} \hat{\alpha}^{-1} \hat{z} \hat{\alpha}+\hat{\alpha}^{-1} \hat{z} \hat{\alpha} \\
T|x, y\rangle & =\ell_{y}|x+1, y+1\rangle+m_{x+1} \frac{\alpha_{x, y}}{\alpha_{x+1, y-1}}|x+2, y\rangle+\frac{\alpha_{x, y}}{\alpha_{x+1, y-1}}|x+1, y-1\rangle
\end{aligned}
$$

Let $Z_{i, j}(n)$ denote the partition function for paths from $s_{i}$ to $e_{j}$ on $\hat{G}_{\min }(n)$.

Lemma 27. We have:

$$
Z_{i, j}(n)=\left\langle j-1, j-1\left|(I-T)^{-1}\right| 1-i, i-1\right\rangle
$$

or equivalently:

$$
Z_{i, j}(n)=\left\langle 0,0\left|\hat{w}^{1-j}(I-T)^{-1} \hat{z}^{1-i}\right| 0,0\right\rangle
$$

Proof. By matching the Definition 26 with the weights of (5.2) (b), we easily see that each step of a path on $\hat{G}_{\text {min }}(n)$ is generated by $T$. More precisely, the quantity $\left\langle u, v\left|T^{k}\right| x, y\right\rangle$ is the sum over all paths on $\hat{G}_{\text {min }}(n)$ of length $k$ starting at the point $(x, y)$ and ending at the point $(u, v)$, of the product of the corresponding network edge weights. The first part of the Lemma follows. For the second part, we note that the adjoint $w^{\dagger}=w^{-1}$, and $w|x, y\rangle=|x+1, y+1\rangle$, so the dual is

$$
\langle x+1, y+1|=\langle x, y| \hat{w}^{\dagger}=\langle x, y| \hat{w}^{-1}
$$

The second formula follows.

Finally, combining Lemma 27 and Theorem 25, we obtain a compact expression for the generalized Lambda-determinant:

\section{Theorem 28.}

$$
|A|_{\lambda, \mu}=\operatorname{det}_{1 \leqslant i, j \leqslant n}\left\langle 0,0\left|\hat{w}^{1-j} \hat{\alpha}\left(I-\hat{w} \hat{\ell}-\hat{w} \hat{m} \hat{\alpha}^{-1} \hat{z} \hat{\alpha}-\hat{\alpha}^{-1} \hat{z} \hat{\alpha}\right)^{-1} \hat{z}^{1-i}\right| 0,0\right\rangle
$$


Proof. By definition, we have

$$
\hat{a}_{\text {min }}(n)=\operatorname{det}_{1 \leqslant i, j \leqslant n} Z_{i, j}(n)=\sum_{\sigma \in S_{n}} \operatorname{sgn}(\sigma) \prod_{j=1}^{n} Z_{\sigma(j), j}(n)
$$

The formula of Theorem 25 includes a prefactor $\prod_{j=1}^{n} a_{n, j}$. Let us absorb the terms $\alpha_{j-1, j-1}=a_{n, j}$ in each of the factors $Z_{\sigma(j), j}(n), j=1,2, \ldots, n$. As $\langle 0,0| \hat{w}^{1-j}=\langle j-1, j-1|$, we have $\alpha_{j-1, j-1}\langle 0,0| \hat{w}^{1-j}=\langle 0,0| \hat{w}^{1-j} \hat{\alpha}$. The theorem follows.

Remark 29. As a non-trivial check of Theorem 28, let us evaluate the determinant in the homogeneous case when $\lambda_{a}=\lambda$ and $\mu_{a}=\mu$ for all $a$, and $a_{i, j}=1$ for all $i, j$. All the operators $\hat{z}, \hat{w}, \hat{\ell}, \hat{m}$ commute, and $\hat{b}=I . Z_{i, j}(n)$ is therefore simply the coefficient of $z^{i-1} w^{j-1}$ of the series:

$$
f_{Z}(z, w)=\frac{1}{1-z-\lambda w-\mu z w}
$$

where the subscript $Z$ stands for the (infinite) matrix with entries $Z_{i, j}(\infty)$. Note that $f_{Z}$ is independent of $n$, which is simply the size of the initial principal minor of $Z$ we are supposed to compute to obtain $|A|_{\lambda, \mu}$. We have the following (infinite size) LU decomposition:

$$
Z=B(1,1)^{t} B(\lambda+\mu, \lambda)
$$

where the infinite matrices $B(\alpha, \beta)$ are upper triangular, with non-zero matrix elements $B(\alpha, \beta)_{i, j}=\left(\begin{array}{l}j \\ i\end{array}\right) \alpha^{i} \beta^{j-i}$ for $j \geqslant i \geqslant 0$, or equivalently with generating functions

$$
f_{B(\alpha, \beta)}(z, w)=\sum_{i, j \geqslant 0} B(\alpha, \beta)_{i, j} z^{i} w^{j}=\frac{1}{1-\beta w-\alpha z w}
$$

The LU decomposition is easily obtained by the convolution formula:

$$
f_{B(1,1)^{t} B(\lambda+\mu, \lambda)}(z, w)=\oint \frac{d t}{2 i \pi t} f_{B(1,1)^{t}}\left(z, t^{-1}\right) f_{B(\lambda+\mu, \lambda)}(t, w) .
$$

where the integral extracts the constant term in $t$. Finally, by triangularity of the factors, the product $Z=B(1,1)^{t} B(\lambda+\mu, \lambda)$, when truncated to the first $n$ rows and columns, truncates to the product of the $n \times n$ truncations of the factors. We deduce that the initial principal $n \times n$ minor is:

$$
|A|_{\lambda, \mu}=\operatorname{det}_{0 \leqslant i, j \leqslant n-1}\left(B(1,1)_{j, i}\right) \operatorname{det}_{0 \leqslant i, j \leqslant n-1}\left(B(\lambda+\mu, \lambda)_{i, j}\right)=(\lambda+\mu)^{n(n-1) / 2},
$$

in agreement with the result of Example 11 for $a_{i}=1$ for all $i$.

\subsection{Transformation into 6V model/ASM: proof of Theorem 2}

By Theorem 25, the generalized Lambda-determinant is expressed as a sum over con-

figurations of $n$ non-intersecting paths on the network $\hat{G}_{\min }(n)$. Any such configuration 
determines entirely the state of the down-pointing triangles labelled by the matrix elements $\left(a_{i, j}\right)_{i, j \in I}$ (we may draw such triangles around $a_{i, 1}, a_{i, n} a_{1, j}$ and $a_{n, j}$ as well). We say that a vertex (resp. edge) is occupied (resp. empty) if a path of the configuration traverses it. This gives rise to seven possible local configurations of the bottom vertex and the three edges of each down-pointing triangle, that we associate with the local configurations of the $6 \mathrm{~V}$ model as follows:
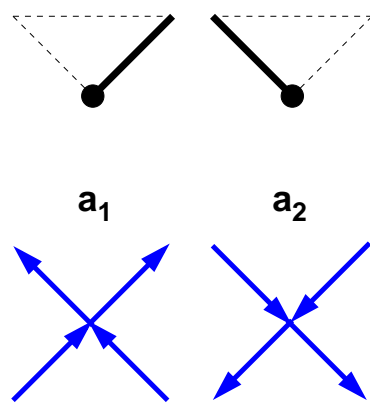
$a_{2}$
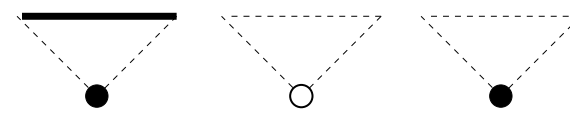

$b_{1}$
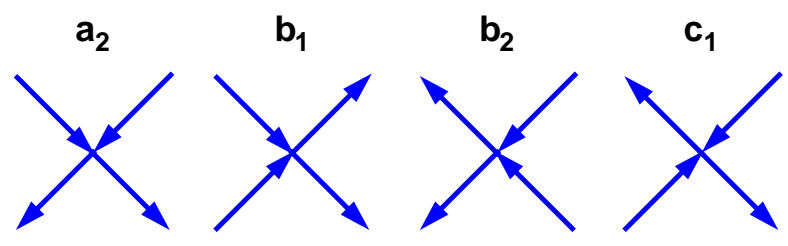

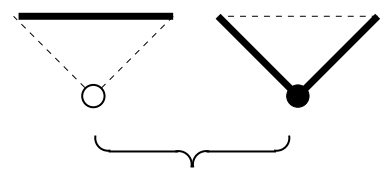

$c_{2}$

where we have represented occupied vertices/edges by filled circles/thick solid lines, and empty ones by empty circles/dashed lines. This is a mapping $2^{m}$ to 1 of the configurations of $n$ non-intersecting paths on $\hat{G}_{\min }(n)$ with a total of $m$ down-pointing triangle configurations among the two on the right to those of the $6 \mathrm{~V}$ model with DWBC on the $n \times n$ grid rotated by $+\pi / 4$, with $m$ vertices of type $c_{2}$. The latter in turn are in bijection with ASMs of size $n \times n$ with $m$ entries equal to -1 , following the rules of (1.1). This is illustrated in the case $n=4, m=1$ below:
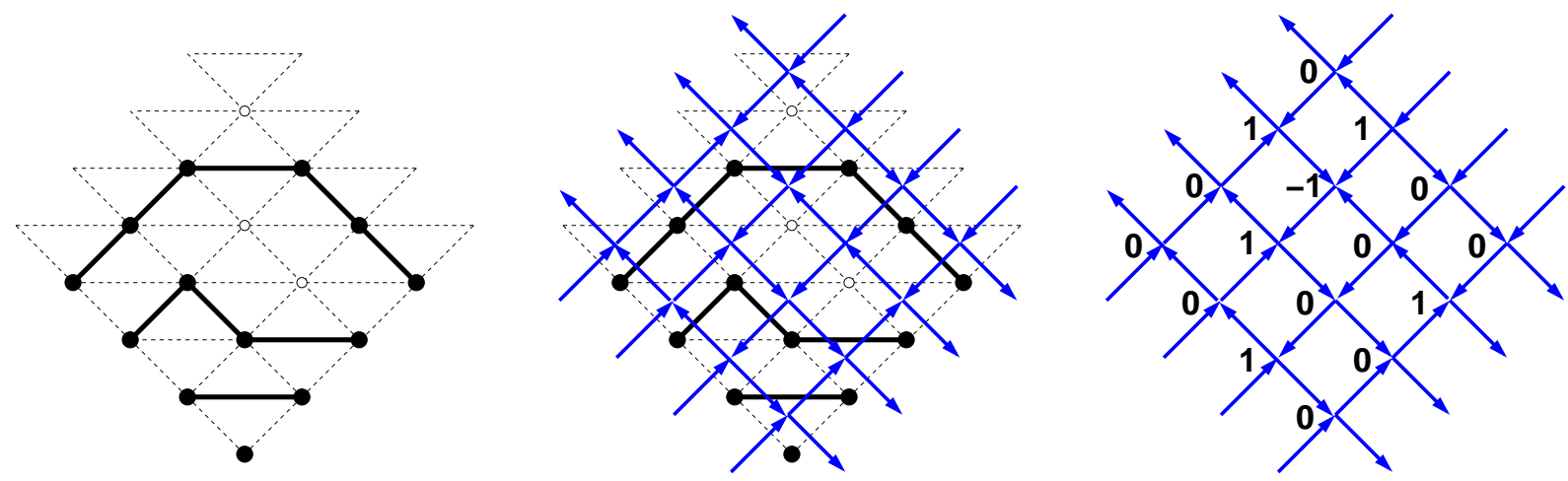

Let us now collect the various weights of the path configurations contributing to $|A|_{\lambda, \mu}$, by distinguishing the dependence on the matrix elements of $A$ (face labels) and the coefficients $\lambda, \mu$ (edge labels). The matrix elements $a_{i, j}$ are the labels of the down-pointing triangular faces. For each face labeled $a$, only four edges of the network $\hat{G}_{\min }(n)$ have weights depending on $a$. These are:

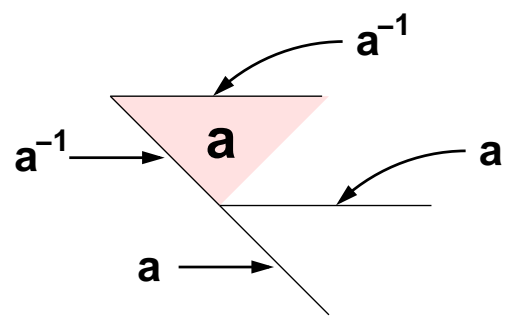


where we have shaded the down-pointing triangle with label $a$, and indicated the $a$ dependence of the four relevant edges. This gives rise to the only following contributions involving the matrix element $a$, depending on the state of occupation of the four above edges, which we list by configuration of the down-pointing triangle:

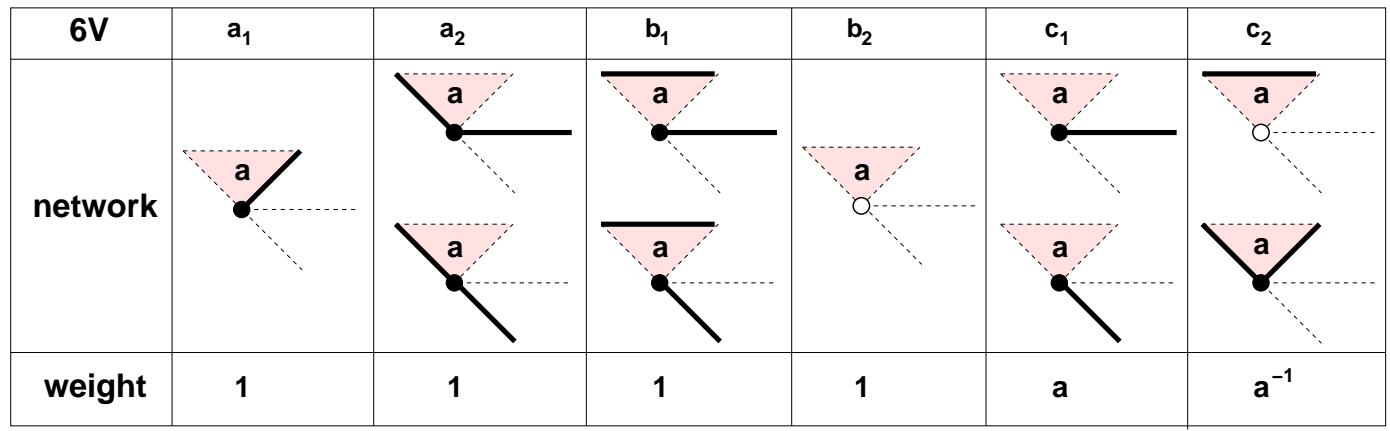

The dependence on the face label $a=a_{i, j}$ is therefore $a^{b}$, where $b=b_{i, j} \in\{0, \pm 1\}$ is the matrix element of the ASM in bijection with the associated $6 \mathrm{~V}$ model configuration. This however is only valid for $i \neq n$, and extra care should be taken with the SE down-pointing triangles. Indeed, their bottom vertex must always be occupied (it is an exit point for the non-intersecting path configuration), and only two configurations may occur: (i) with the top edge occupied (6V configuration $b_{1}$ ) or (ii) with the left diagonal edge occupied (6V configuration $a_{2}$ ). In both cases, the occupied edge receives a weight $a^{-1}$ :
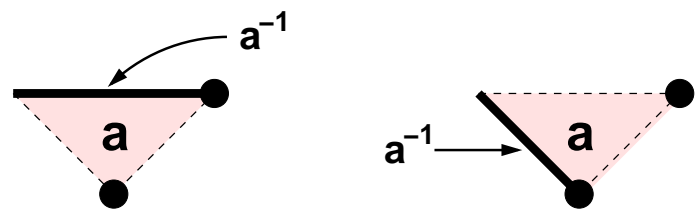

However, recall that the formula for the generalized Lambda-determinant of Theorem 25 contains a prefactor $\prod_{i=1}^{n} a_{n, i}$ which is the product over the labels of the SE downpointing triangles. We may therefore absorb this prefactor in a redefinition of the weights of horizontal and left diagonal edges adjacent to the SE faces, namely by multiplying them by the face label $a$. The resulting network $G_{\min }^{\prime}(n)$ and the $n \times n$ initial principal minor $a_{\min }^{\prime}(n)$ of its matrix are such that

$$
|A|_{\lambda, \mu}=a_{\min }^{\prime}(n) .
$$

Note that this latter transformation of the network into $G_{\min }^{\prime}(n)$ corresponds exactly to the insertion of the $\hat{\alpha}$ operator in Theorem 28. According to the table (5.6), each configuration of $n$ non-intersecting paths on the network $G_{m i n}^{\prime}(n)$ that contributes to $a_{m i n}^{\prime}(n)$ receives a weight $\prod_{i, j} a_{i, j}^{b_{i, j}}$ where $b_{i, j}$ is the ASM in bijection with the $6 \mathrm{~V}$ configuration associated to the path configuration.

Finally, the dependence of the edge weights on the coefficients $\lambda_{a}, \mu_{a}$ is clear from $(5.2)(b)$ : around the down-pointing triangle with label $a_{i, j}$, the horizontal edge receives 
the weight $\mu_{i+j-n-1}$, while the left diagonal edge receives the weight $\lambda_{j-i}$. This gives the total weights

\begin{tabular}{|c|c|c|c|c|c|c|}
\hline $6 \mathrm{~V}$ & $a_{1}$ & $a_{2}$ & $b_{1}$ & $b_{2}$ & $c_{1}$ & $c_{2}$ \\
\hline network & & & C & $r$ & . & a \\
\hline weight & $\lambda$ & 1 & $\mu$ & 1 & $\mathbf{a}$ & $\mu \mathbf{a}^{-1}$ \\
\hline
\end{tabular}

where for $a=a_{i, j}, \lambda=\lambda_{j-i}$ and $\mu=\mu_{i+j-n-1}$, for all $i, j=1,2, \ldots, n$.

Starting from the expression (5.7), we may rearrange the configurations of paths with a total of $m$ triangle configurations of type $c_{2}$ say in positions $\left(i_{1}, j_{1}\right),\left(i_{2}, j_{2}\right), \ldots,\left(i_{m}, j_{m}\right)$ (and a total of $m+n$ triangle configurations of type $c_{1}$ in fixed positions) into a $2^{m}$-tuple, in bijection with the unique ASM with exactly $m$ entries -1 at the same positions (and $m+n$ entries equal to 1 at the same positions). Summing over the weights for such a $2^{m}$-tuple yields an overall factor of

$$
\prod_{r=1}^{m}\left(\lambda_{j_{r}-i_{r}}+\mu_{i_{r}+j_{r}-n-1}\right)
$$

according to the table (5.8). Collecting all the other weights, we may rewrite $|A|_{\lambda, \mu}$ as a sum over $n \times n$ ASMs $B$, with the weights of the form $\prod_{i, j \in I} v_{i, j}\left(a_{i, j}, b_{i, j} \mid \lambda_{j-i}, \mu_{i+j-n-1}\right)$ where

$$
v_{i, j}(a, b \mid \lambda, \mu)=a^{b} \times\left\{\begin{array}{cc}
\lambda & \text { if } \mathcal{C}_{i, j}(B)=a_{1} \\
\mu & \text { if } \mathcal{C}_{i, j}(B)=b_{1} \\
\lambda+\mu & \text { if } \mathcal{C}_{i, j}(B)=c_{2} \\
1 & \text { otherwise }
\end{array}\right.
$$

This completes the proof of Theorem 2 .

\section{Discussion and conclusion}

In this paper, we have defined the generalized Lambda-determinant of any square matrix $A$. Via Theorem 2 this quantity may be reexpressed in terms of weighted configurations of the $6 \mathrm{~V}$-DWBC model on a square grid of the same size as $A$, in bijection with ASMs of the same size.

\subsection{Properties}

Let us show how the formula of Theorem 2 may be applied to give an alternative proof of Proposition 12. Notations are as in Proposition 12.

We simply have to examine how the $6 \mathrm{~V} / \mathrm{ASM}$ configurations contributing to the formula (1.2) are changed when we change $A \rightarrow \varphi(A)$, namely (i) rotate the matrix $A$ by a clockwise quarter-turn $-\pi / 2(\varphi=\sigma)$ or (ii) transpose the matrix $A$, namely reflect 
it w.r.t. to its diagonal $(\varphi=\tau)$. We have the following action of $\varphi$ on the $6 \mathrm{~V}$-DWBC configurations $\mathcal{C}$, compatible with the transformation $B \rightarrow \varphi(B)$ for any $n \times n$ ASM $B$ :

Lemma 30. Let $\varphi \in\{\sigma, \tau\}$ act on configurations $\mathcal{C}$ of the $n \times n$ grid $6 \mathrm{~V}$-DWBC model as follows: $\varphi(\mathcal{C})$ is obtained by applying directly the transformation $\varphi$ to the arrow configuration (rotation by $-\pi / 2$ for $\varphi=\sigma$, reflection w.r.t. the diagonal for $\varphi=\tau$ ), and then performing a global flip of all the edge orientations. Then we have for all $n \times n$ ASM B:

$$
\varphi(\mathcal{C}(B))=\mathcal{C}(\varphi(B))
$$

Proof. Note first that the global sign flip reinstates the correct orientations of external edges to restore DWBC boundary conditions. Under the transformation $\varphi$, the six vertex configurations are mapped as follows:

$\sigma\left(a_{1}\right)=b_{2} \quad \sigma\left(b_{1}\right)=a_{1} \quad \sigma\left(a_{2}\right)=b_{1} \quad \sigma\left(b_{2}\right)=a_{2} \quad \sigma\left(c_{1}\right)=c_{1} \quad \sigma\left(c_{2}\right)=c_{2}$ $\tau\left(a_{1}\right)=a_{1} \quad \tau\left(b_{1}\right)=b_{2} \quad \tau\left(a_{2}\right)=a_{2} \quad \tau\left(b_{2}\right)=b_{1} \quad \tau\left(c_{1}\right)=c_{1} \quad \tau\left(c_{2}\right)=c_{2}$

As both $c_{1}$ and $c_{2}$ are invariant under $\varphi \in\{\sigma, \tau\}$, the ASM associated to the configuration $\varphi(\mathcal{C})$ is simply $\varphi(B)$, and the Lemma follows.

Let us now evaluate $|\varphi(A)|_{\varphi(\lambda), \varphi(\mu)}$. Using the formula (1.2), the weight $w$ reads:

$$
w_{i, j}(\varphi(A), B ; \varphi(\lambda), \varphi(\mu))=\left(\varphi(A)_{i, j}\right)^{b_{i, j}} \times\left\{\begin{array}{cl}
\varphi(\lambda)_{j-i} & \text { if } \mathrm{C}(B)_{i, j}=a_{1} \\
\varphi(\mu)_{i+j-n-1} & \text { if } \mathrm{C}(B)_{i, j}=b_{1} \\
\varphi(\lambda)_{j-i}+\varphi(\mu)_{i+j-n-1} & \text { if } \mathrm{C}(B)_{i, j}=c_{2} \\
1 & \text { otherwise }
\end{array}\right.
$$

Let us perform the change of summation variables $B=\varphi(C)$. For $\varphi=\sigma, \tau$ respectively, this gives weights:

$$
\begin{gathered}
w_{i, j}(\sigma(A), \sigma(C) ; \sigma(\lambda), \sigma(\mu))=\left(a_{n+1-j, i}\right)^{c_{n+1-j, i}} \times\left\{\begin{array}{cc}
\mu_{i-j} & \text { if } \mathrm{C}(C)_{n+1-j, i}=b_{1} \\
\lambda_{i+j-n-1} & \text { if } \mathrm{C}(C)_{n+1-j, i}=a_{2} \\
\mu_{i-j}+\lambda_{i+j-n-1} & \text { if } \mathrm{C}(C)_{n+1-j, i}=c_{2} \\
1 & \text { otherwise }
\end{array}\right. \\
w_{i, j}(\tau(A), \tau(C) ; \tau(\lambda), \tau(\mu))=\left(a_{j, i}\right)^{c_{j, i}} \times\left\{\begin{array}{cc}
\lambda_{i-j} & \text { if } \mathrm{C}(C)_{j, i}=a_{1} \\
\mu_{i+j-n-1} & \text { if } \mathrm{C}(C)_{j, i}=b_{2} \\
\lambda_{i-j}+\mu_{i+j-n-1} & \text { if } \mathrm{C}(C)_{j, i}=c_{2} \\
1 & \text { otherwise }
\end{array}\right.
\end{gathered}
$$

Equivalently, we may write:

$$
\begin{array}{r}
w_{j, n+1-i}(\sigma(A), \sigma(C) ; \sigma(\lambda), \sigma(\mu))=\left(a_{i, j}\right)^{c_{i, j}} \times\left\{\begin{array}{cc}
\mu_{i+j-n-1} & \text { if } \mathrm{C}(C)_{i, j}=b_{1} \\
\lambda_{j-i} & \text { if } \mathrm{C}(C)_{i, j}=a_{2} \\
\lambda_{j-i}+\mu_{i+j-n-1} & \text { if } \mathrm{C}(C)_{i, j}=c_{2} \\
1 & \text { otherwise }
\end{array}\right. \\
w_{j, i}(\tau(A), \tau(C) ; \tau(\lambda), \tau(\mu))=\left(a_{i, j}\right)^{c_{i, j}} \times\left\{\begin{array}{cl}
\lambda_{j-i} & \text { if } \mathrm{C}(C)_{i, j}=a_{1} \\
\mu_{i+j-n-1} & \text { if } \mathrm{C}(C)_{i, j}=b_{2} \\
\lambda_{j-i}+\mu_{i+j-n-1} & \text { if } \mathrm{C}(C)_{i, j}=c_{2} \\
1 & \text { otherwise }
\end{array}\right.
\end{array}
$$




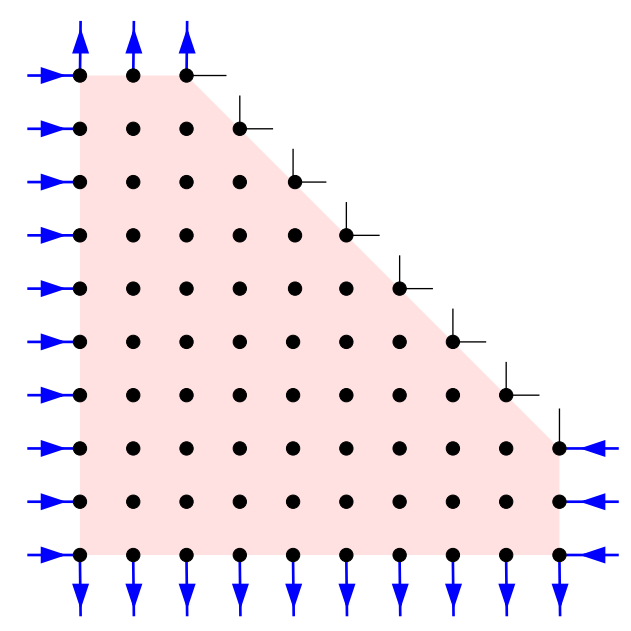

(a)

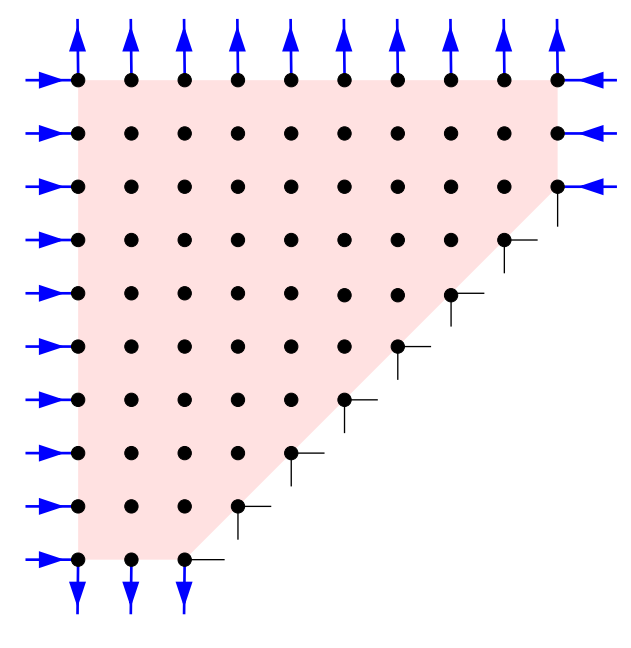

(b)

Figure 1: The sub-configuration of a $6 \mathrm{~V}-\mathrm{DWBC}$ configuration $\mathcal{C}$ situated (a) under a diagonal (b) above an anti-diagonal. We have marked the edges of the diagonal border, respectively equal to the $\mathrm{N}$ and E edges (a) and $\mathrm{S}$ and E edges (b).

These two expressions match $w_{i, j}(A, C ; \lambda, \mu)$ provided the $6 \mathrm{~V}$ weights in the definition are changed into respectively (i) $a_{1} \rightarrow a_{2}$ and (ii) $b_{1} \rightarrow b_{2}$.

Proposition 12 follows from the fact that the formula (1.2) remains valid if we change $a_{1} \rightarrow a_{2}$ and/or $b_{1} \rightarrow b_{2}$ in the definition of $w$. This in turn is the consequence of the following:

Lemma 31. For any $6 \mathrm{~V}-D W B C$ configuration $\mathcal{C}$, we have for all a:

$$
\begin{aligned}
& \mid\left\{i \in\{1,2, \ldots, n\} \text { such that } \mathcal{C}_{i, i+a}=a_{1}\right\}|=|\left\{i \in\{1,2, \ldots, n\} \text { such that } \mathcal{C}_{i, i+a}=a_{2}\right\} \mid \\
& \mid\left\{i \in\{1,2, \ldots, n\} \text { such that } \mathcal{C}_{i, a-j}=b_{1}\right\}|=|\left\{i \in\{1,2, \ldots, n\} \text { such that } \mathcal{C}_{i, a-j}=b_{2}\right\} \mid
\end{aligned}
$$

in other words there are as many $a_{1}$ configurations as $a_{2}$ along each parallel to the diagonal, and there are as many $b_{1}$ configurations as $b_{2}$ along each parallel to the anti-diagonal.

Proof. Let us consider a diagonal $(i, i+a)_{1 \leqslant i \leqslant n-a}$ of $\mathcal{C}$, and the sub-configuration situated under the line $(i, i+a-1)$ (see Fig.1 (a)). This configuration has entering edges on its left and possibly right vertical borders, and outgoing edges along its bottom and possibly top horizontal borders, in equal numbers. By the neutrality condition at each vertex (there are as many entering as outgoing edges), we deduce that there are as many entering as outgoing edges along the diagonal border. These are the $\mathrm{N}$ and E edges of the vertices of the diagonal border. Inspecting the vertices on (1.1) we see that all vertices have opposite orientations of their $\mathrm{N}$ and E edges, except for $a_{1}$ and $a_{2}$ who have respectively two outgoing and two entering $\mathrm{N}$ and $\mathrm{E}$ edges. We deduce that these occur in the same number to guarantee that as many edges are entering and outgoing along the diagonal border. The argument may be repeated for anti-diagonals by considering the 
sub-configuration situated above the anti-diagonal $(i, a+1-i)$ (see Fig.1 (b)). In that case, we must inspect the $\mathrm{S}$ and $\mathrm{E}$ edges of the vertices of (1.1), and we note that only $b_{1}$ and $b_{2}$ have $\mathrm{S}$ and $\mathrm{E}$ edges pointing both out or in, therefore they must be in equal number along the diagonal border. The Lemma follows.

\subsection{The generalized Lambda-determinant in terms of ASM}

Theorem 2 expresses the generalized Lambda-determinant in terms of 6V-DWBC configurations. Let us reexpress this quantity purely in ASM terms. To this end, we must better explain the inversion number of any ASM $B$. The contributions to $\operatorname{Inv}(B)=$

$\sum_{i<j} \quad k<\ell=b_{j, k} b_{i, \ell}$ are obtained from rectangular submatrices of $B_{[i, j]}^{[k, \ell]}$ of $B$, obtained by retaining only the intersection between rows $(i, i+1, \ldots, j)$ and columns $(k, k+1, \ldots, \ell)$ in $B$. To produce a non-trivial contribution to $\operatorname{Inv}(B)$ the rectangle must have non-zero elements in position $(j, k)(\mathrm{SW})$ and $(i, \ell)(\mathrm{NE})$. Let us now concentrate on the element in position $(i, k)(\mathrm{NW})$. Let $h, v$ denote respectively the first non-zero element of $B$ on row $i$ to the right of $b_{i, k}$ and on column $k$ below $b_{i, k}$. The simplest way of thinking about the bijection from ASM to $6 \mathrm{~V}$-DWBC is that along rows edges are oriented from the entries -1 to the entries 1 and get reversed at the first and last 1, with opposite rules along columns. Assuming $b_{i, k}=0$, this gives the following 4 possibilities: (i) $(h, v)=(1,1)$ : then $\mathrm{C}(B)_{i, k}=a_{1}$; (ii) $(h, v)=(-1,1)$ : then $\mathrm{C}(B)_{i, k}=b_{2}$; (iii) $(h, v)=(1,-1)$ : then $\mathcal{C}(B)_{i, k}=b_{1}$; (iv) $(h, v)=(-1,-1)$ : then $\mathcal{C}(B)_{i, k}=a_{2}$. When computing contributions to $\operatorname{Inv}(B)$, we may first fix the NW corner of rectangles we sum over, and finally sum over the position $(i, k)$ of that corner. In cases (ii-iv), for fixed $(i, k)$ the sum gives trivially zero, as there are as many 1's and -1 either on the row or the column of $(i, k)$ or both. So only the case (i) contributes a total of +1 to $\operatorname{Inv}(B)$. Moreover, if $b_{i, k} \neq 0$, only cases (i) and (iv) survive, with respectively $b_{i, k}=-1$ and $b_{i, k}=1$. However for the same reason as before, the case (iv) does not contribute to $\operatorname{Inv}(B)$ when we sum over the rectangles with fixed NW corner $b_{i, k}$. We are left with only the case $(\mathrm{i})$, for which $\mathrm{C}(B)_{i, j}=c_{2}$, and the sum over rectangles contributes a total of +1 to $\operatorname{inv}(B)$.

To summarize, terms contributing +1 to $\operatorname{Inv}(B)$ may be associated bijectively to the $a_{1}$ and $c_{2}$ configurations in $\mathcal{C}(B)$. So we have $\operatorname{Inv}(B)-\#(-1)_{B}=\#\left(a_{1}\right)$, the total number of $a_{1}$ vertices in $\mathrm{C}(B)$. Moreover, the vertices equal to $a_{1}$ in $\mathrm{C}(B)$ correspond bijectively to the entries 0 of $B$, such that the first non-zero elements in $B$ in their row to the right and in their column below are both 1 .

We may repeat the above analysis with the matrix $\sigma(B)$, rotated by $-\pi / 2$. What plays the role of $a_{1}$ after rotation are the vertices $b_{1}$ before rotation (see previous subsection), and $c_{2}$ 's remain unchanged. We deduce that $\operatorname{Inv}(\sigma(B))-\#(-1)_{B}=\#\left(b_{1}\right)$, the total number of $b_{1}$ vertices in $\mathrm{C}(B)$. Moreover the vertices equal to $b_{1}$ in $\mathcal{C}(B)$ correspond bijectively to the entries 0 of $B$, such that the first non-zero elements in $B$ in their row to the right and in their column above are both 1 .

Definition 32. For each fixed diagonal $\delta_{a}(B)=\left\{b_{i, j} \mid j-i=a\right\}$ for $2-n \leqslant a \leqslant n-2$, let us denote by $\mathrm{I}_{a}(B)$ the total number of entries 0 such that the first non-zero entries to the right and below are both 1 . For each fixed anti-diagonal $\alpha_{b}=\left\{b_{i, j} \mid i+j=b\right\}$ for 
$2-n \leqslant b \leqslant n-2$ let $\mathrm{I}_{b}^{\prime}(B)$ denote the total number of entries 0 such that the first non-zero entries to the right and above are both 1 . Finally, let $\#(-1)_{B}(i, j)=\delta_{b_{i, j},-1}$.

We have the following generalized Lambda-determinant expression:

Theorem 33. The generalized Lambda-determinant of any $n \times n$ matrix $A$ reads:

$$
|A|_{\lambda, \mu}=\sum_{n \times n A S M B} \prod_{a=2-n}^{n-2} \lambda_{a}^{\mathrm{I}_{a}(B)} \mu_{a}^{\mathrm{I}_{a}^{\prime}(B)} \prod_{i, j=1}^{n} a_{i, j}^{b_{i, j}}\left(\lambda_{j-i}+\mu_{i+j-n-1}\right)^{\#(-1)_{B}(i, j)}
$$

\subsection{The general solution of the $T$-system with coefficients}

In this paper, we have concentrated on special initial conditions for the $T$-system (3.2), namely such that $T_{i, j, 0}=1$ and $T_{i, j, 1}=a_{\underline{j-i+n+1}}, \underline{i+j+n+1}$. Starting from the general $T$ system solution of Theorem 20, where both $T_{i, j, 0}^{2}$ and $T_{i, j, 1}^{2}$ are assigned arbitrary initial values $t_{i, j}=T_{i, j, \epsilon_{i, j, n}}$, we may repeat the steps we took to modify the initial network $G_{\min }(n)$ into a subset of the directed triangular lattice. The resulting network (which we still denote by $\hat{G}_{\min }(n)$, with associated minor $\hat{a}_{\text {min }}(n)$ by a slight abuse of notation) has the following edge weights around each up-pointing triangle:

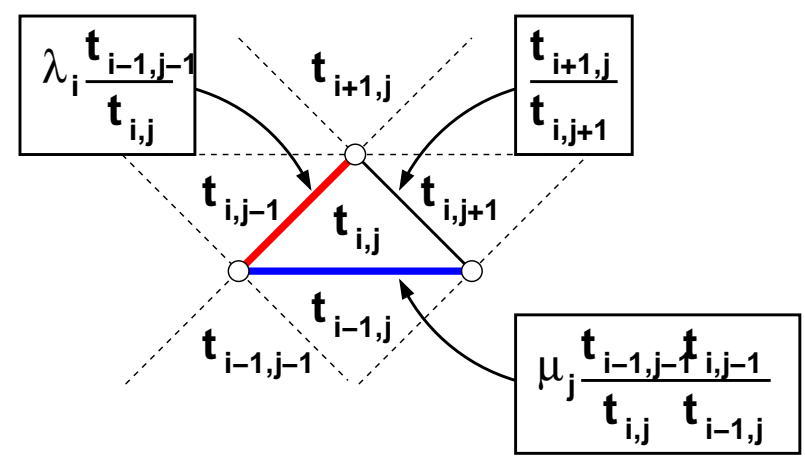

and we have

$$
T_{0,0, n}=\hat{a}_{m i n}(n) \prod_{j=1}^{n} t_{j-n, j-1}=a_{m i n}^{\prime}(n)
$$

where the last transformation to $G_{\min }^{\prime}(n)$ consists in absorbing the $t_{j-n, j-1}$ in the SE horizontal and diagonal edge weights adjacent to the endpoints of the paths. Noting that the initial values $T_{i, j, 1}$ are the face labels of the down-pointing triangles, while the $T_{i, j, 0}$ are those of the up-pointing triangles, we may summarize the dependence of the edge weights on the face labels of the down-pointing and up-pointing triangles as follows:
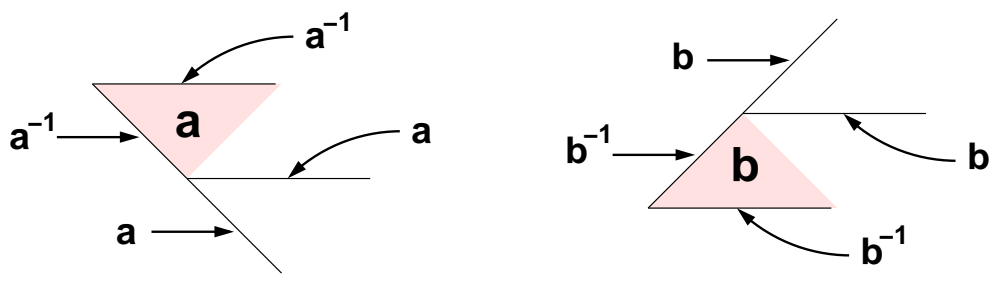
where $a, b=t_{i, j}$ for some $i, j$. Combining these with the weights of down-pointing triangles (5.6), we note that the weights acquire a simpler form if we transfer the $\lambda$ and $\mu$ parameters from the weights of down-pointing triangles to those of up-pointing ones, resulting in:

\begin{tabular}{|c|c|c|c|c|c|c|c|}
\hline network & $\mathbf{a}$ & $\mathbf{a}$ & $\mathbf{a}$ & $\mathbf{a}$ & $\mathbf{a}$ & $\mathbf{a}$ & $\mathbf{a}$ \\
\hline weight & $\mathbf{1}$ & $\lambda$ & $\mu$ & $\mathbf{1}$ & $\mathbf{a}$ & $\mu \mathbf{a}^{-1}$ & $\lambda \mathbf{a}^{-1}$ \\
\hline network & $\mathbf{a}$ & $\mathbf{a}$ & $\mathbf{a}$ & $\mathbf{a}$ & $\mathbf{a}$ & $\mathbf{a}$ & $\mathbf{a}$ \\
\hline weight & $\mathbf{1}$ & $\mathbf{1}$ & $\mathbf{1}$ & $\mathbf{1}$ & $\mathbf{a}$ & $\mathbf{a}^{-1}$ & $\mathbf{a}^{-1}$ \\
\hline
\end{tabular}

where for $a=t_{i, j}$, we have $\lambda=\lambda_{i}$ and $\mu=\mu_{j}$.

Remark 34. We have associated a $6 \mathrm{~V}$-DWBC configuration of size $n$ to each configuration of $n$ non-intersecting paths on $G_{\min }^{\prime}(n)$ from $S W$ to $S E$, by the local rules (5.5) associating $6 V$ vertices to each down-pointing triangle configuration. We could have done the same for up-pointing triangles as well, by using the same rules on the reflected up-pointing triangles w.r.t. their horizontal edge. More precisely, this would yield a $6 \mathrm{~V}$-DWBC' configuration of size $n-1$ where the prime refers to the fact that the boundary condition has opposite convention (horizontal external edges point out of the grid, while vertical ones point into the grid). Flipping all edge orientations, this becomes a $6 \mathrm{~V}$-DWBC configuration of size $n-1$. So each configuration of non-intersecting paths on $G_{\text {min }}^{\prime}(n)$ that contribute to $a_{\min }^{\prime}(n)$ give rise naturally to a pair $(B, C)$ of $A S M$ s of respective sizes $n$ and $n-1$. Such pairs are called compatible pairs in the literature. However, these pairs do not play any role in our fully inhomogeneous T-system solution, as weights are attached only to such compatible pairs and cannot be disentangled nicely. In particular there is no resummation of the non-intersecting lattice path weights into weights only pertaining to the ASMs, as opposed to the case where $T_{i, j, 0}=1$ of Theorem 2, where the second ASM plays no role.

Example 35. We consider $n=3$. We have the initial data assignments (represented in the $(i, j)$ plane):

$$
\begin{array}{rlrl}
T_{2,0,1} & =t_{2,0} \\
T_{1,-1,1} & =t_{1,-1} & T_{1,0,0} & =t_{1,0} \\
T_{0,-2,1}=t_{0,-2} & T_{1,1,1} & =t_{1,1} \\
T_{0,-1,0} & =t_{0,-1} & T_{0,0,1} & =t_{0,0} \\
T_{-1,-1,1} & =t_{-1,-1} & T_{0,1,0} & =t_{0,1} \\
T_{-1,0,0} & =t_{-1,0} & T_{-1,1,1} & =t_{-1,1} \\
T_{-2,0,1} & =t_{-2,0}
\end{array}
$$

There are 8 distinct configurations of the 3 non-intersecting paths on $G_{\min }^{\prime}(3)$, contributing to $a_{\text {min }}^{\prime}(3)$ (we have indicated the by small squares the centers of the $n^{2}=9$ down-pointing triangles and of the $(n-1)^{2}=4$ up-pointing ones, corresponding to labels $t_{i, j}$ with $(i, j) \in$ 
$D_{0,0,3}$, on the first configuration (a)):

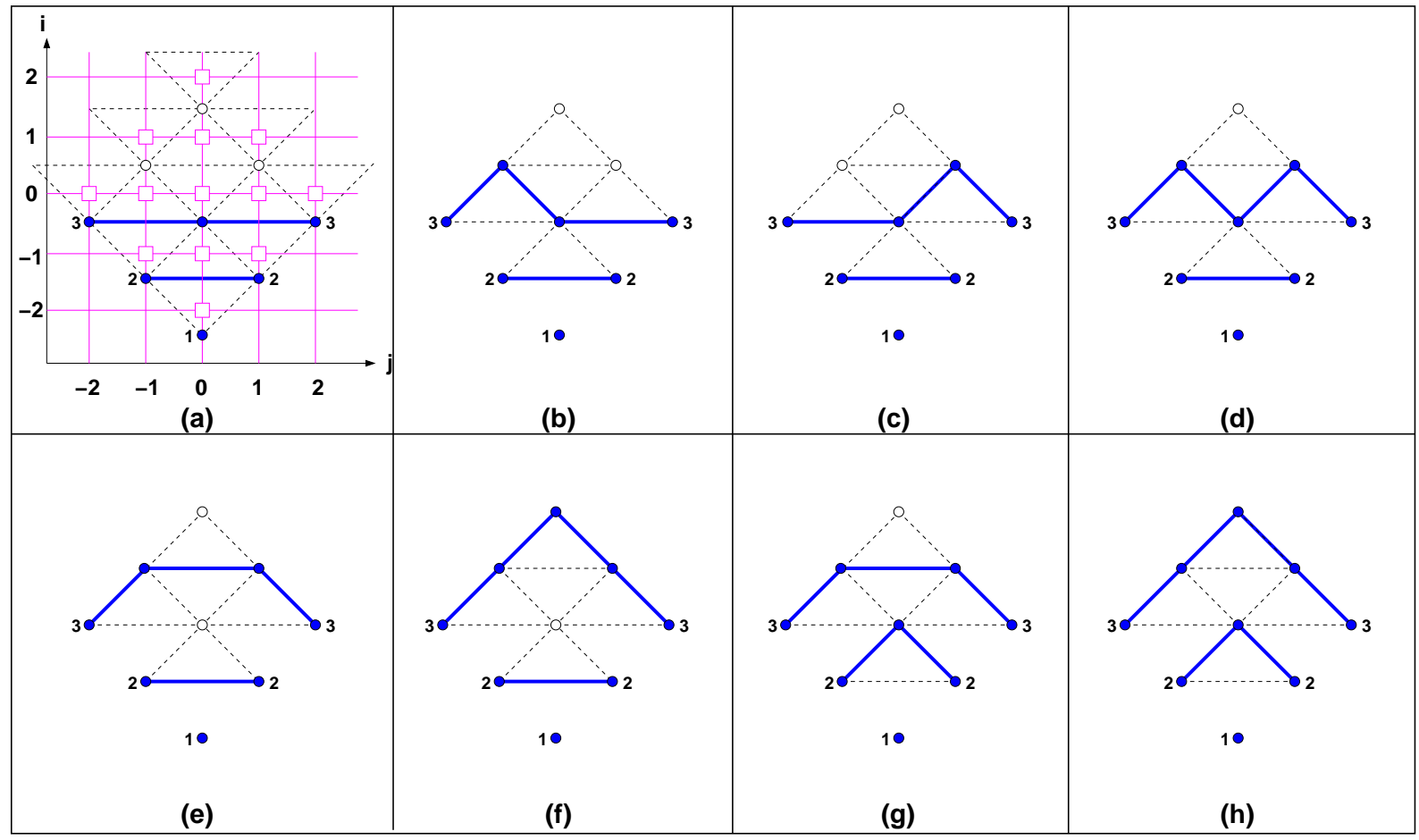

The corresponding weights read:

$$
\begin{aligned}
& w_{(a)}=\mu_{-1} \mu_{0} \mu_{1} \frac{t_{0,-2}, t_{0,0}, t_{0,2}}{t_{0,-1} t_{0,1}} \quad w_{(b)}=\lambda_{0} \mu_{0} \mu_{1} \frac{t_{-1,-1} t_{1,-1} t_{0,2}}{t_{0,-1} t_{0,1}} \quad w_{(c)}=\mu_{-1} \mu_{0} \lambda_{0} \frac{t_{0,-1} t_{-1,1} t_{1,1}}{t_{0,-1} t_{0,1}} \\
& w_{(d)}=\lambda_{0}^{2} \mu_{0} \frac{t_{-1,-1} t_{-1,1} t_{1,-1} t_{1,1}}{t_{0,-1} t_{0,1} t_{0,0}} \quad w_{(e)}=\lambda_{0} \mu_{0}^{2} \frac{t_{-1,-1,-1,1} t_{1,-1} t_{1,1}}{t_{-1,0} t_{1,0} t_{0,0}} \quad w_{(f)}=\mu_{0} \lambda_{0} \lambda_{1} \frac{t_{-1,-1} t_{1,1,1} t_{2,0}}{t_{-1,0} t_{1,0}} \\
& w_{(g)}=\lambda_{-1} \lambda_{0} \mu_{0} \frac{t_{1,-1} t_{1,1} t_{-2,0}}{t_{-1,0}} \quad w_{(h)}=\lambda_{-1} \lambda_{0} \lambda_{1} \frac{t_{-1,-1, t 1,1-2,0}}{t_{-1,0} t_{1,0}}
\end{aligned}
$$

The corresponding solution of the T-system is:

$$
\begin{aligned}
T_{0,0,3}= & \mu_{-1} \mu_{0} \mu_{1} \frac{t_{0,-2}, t_{0,0}, t_{0,2}}{t_{0,-1} t_{0,1}}+\lambda_{0} \mu_{0} \mu_{1} \frac{t_{-1,-1} t_{1,-1} t_{0,2}}{t_{0,-1} t_{0,1}}+\mu_{-1} \mu_{0} \lambda_{0} \frac{t_{0,-1} t_{-1,1} t_{1,1}}{t_{0,-1} t_{0,1}} \\
& +\lambda_{0}^{2} \mu_{0} \frac{t_{-1,-1} t_{-1,1} t_{1,-1} t_{1,1}}{t_{0,-1} t_{0,1} t_{0,0}}+\lambda_{0} \mu_{0}^{2} \frac{t_{-1,-1} t_{-1,1} t_{1,-1} t_{1,1}}{t_{-1,0} t_{1,0} t_{0,0}}+\mu_{0} \lambda_{0} \lambda_{1} \frac{t_{-1,-1} t_{-1,1} t_{2,0}}{t_{-1,0} t_{1,0}} \\
& +\lambda_{-1} \lambda_{0} \mu_{0} \frac{t_{1,-1} t_{1,1} t_{-2,0}}{t_{-1,0} t_{1,0}}+\lambda_{-1} \lambda_{0} \lambda_{1} \frac{t_{-1,-1} t_{-1,1} t_{-2,0}}{t_{-1,0} t_{1,0}}
\end{aligned}
$$

If we take $t_{ \pm 1,0}=t_{0, \pm 1}=1$, we recover the formula of Theorem 2:

$$
\begin{aligned}
T_{0,0,3}= & \mu_{-1} \mu_{0} \mu_{1} t_{0,-2}, t_{0,0}, t_{0,2}+\lambda_{0} \mu_{0} \mu_{1} t_{-1,-1} t_{1,-1} t_{0,2}+\mu_{-1} \mu_{0} \lambda_{0} t_{0,-1} t_{-1,1} t_{1,1} \\
& +\mu_{0} \lambda_{0} \lambda_{1} t_{-1,-1} t_{-1,1} t_{2,0}+\lambda_{-1} \lambda_{0} \mu_{0} t_{1,-1} t_{1,1} t_{-2,0}+\lambda_{-1} \lambda_{0} \lambda_{1} t_{-1,-1} t_{-1,1} t_{-2,0} \\
& +\lambda_{0} \mu_{0}\left(\lambda_{0}+\mu_{0}\right) \frac{t_{-1,-1} t_{-1,1} t_{1,-1} t_{1,1}}{t_{0,0}}
\end{aligned}
$$


where the sum extends over the 7 ASM of size 3. Finally, for $t_{i, j}=1$ for all $i, j$ and $\lambda_{i}=\mu_{i}=q^{i}$ we have:

$$
T_{0,0,3}=4+2\left(q+q^{-1}\right)=\left(1+q^{-1}\right)\left(1+q^{0}\right)\left(1+q^{1}\right)
$$

in agreement with Theorem 13 for $n=3$.

\subsection{Conclusion and perspectives}

A number of questions remain to be answered concerning this new deformation of the determinant.

The partition function of the $6 \mathrm{~V}-\mathrm{DWBC}$ model at its free fermion point $\Delta=0$, $q=e^{i \pi / 2}$, corresponds to the 2-enumeration of ASM, with a weight 2 per entry -1 , and matches the Lambda-determinant, with all $\lambda_{a}=\mu_{b}=1$. However, the 6V-DWBC model is known to have a non-trivial deformation involving $2 n$ spectral parameters say $z_{i}$ per row $i$ and $w_{j}$ per column $j$ of the square lattice $n \times n$ grid. Our generalized Lambda-determinant introduces $2 n-2$ other deformation parameters, which are attached to diagonals and antidiagonals of the same square grid. It would be interesting to mix the two deformations and see if we can obtain some new information on ASMs by the process.

Another question concerns the limit shape. It is known that the $6 \mathrm{~V}$-DWBC model at its free fermion point is also in bijection with the domino tilings of the Aztec diamond [8], for which an arctic circle theorem exists, namely there exists a limiting curve (a circle) separating frozen tiling phases from entropic tiling in the limit of large grid and small mesh size. It is easy to work out the homogeneous deformation of this curve when $\lambda_{a}=\lambda$ and $\mu_{b}=\mu$ for all $a, b$. The simplest way of computing the artic curve in our model (in the case of a matrix $A$ with entries $a_{i, j}=1$ ) is by analyzing the singularities of the generating function of the density $\rho_{i, j, k}$ defined as

$$
\rho_{i, j, k}=\left.\frac{\partial \log T_{i, j, k}}{\partial t_{0,0}}\right|_{t_{i, j}=1 ; i, j \in \mathbb{Z}}
$$

where $T_{i, j, k}$ is the solution of the T-system (3.2) with initial data (4.2). Defining $\rho(X, Y, Z)=\sum_{k \geqslant 1 ; i, j \in \mathbb{Z}} \rho_{i, j, k} X^{i} Y^{j} Z^{k}$ we find, by differentiating the T-system relation and evaluating the result at $t_{i, j}=1$ :

$$
\rho(X, Y, Z)=\frac{Z}{\left(1+Z^{2}-Z\left(\frac{\lambda}{\lambda+\mu}\left(X+X^{-1}\right)+\frac{\mu}{\lambda+\mu}\left(Y+Y^{-1}\right)\right)\right.}
$$

Following [13], we find that in the coordinates $(x, y)$ obtained as limit of $\left(\frac{i}{k}, \frac{j}{k}\right)$, for $(i, j) \in$ $D_{i, j, k}$, of a $k \times k 6 \mathrm{~V}$-DWBC grid rotated by $+\pi / 4$, the singular curve is the following ellipse:

$$
x^{2}\left(1+\frac{\lambda}{\mu}\right)+y^{2}\left(1+\frac{\mu}{\lambda}\right)=1
$$

It would be interesting to see how this is affected by inhomogeneous parameters $\lambda_{a}, \mu_{a}$. In the particular example $\lambda_{i}=\mu_{i}=q^{i}$ of Section 3.3, we find the following equation for 
$\rho(X, Y, Z):$

$$
\left(1+Z^{2}-Z\left(X+X^{-1}\right)\right) \rho(X, Y, Z)+\left(1+Z^{2}-Z\left(q Y+q^{-1} Y^{-1}\right)\right) \rho\left(q^{-1} X, q Y, Z\right)=2 Z
$$

For generic $q$ (not a root of unity) the function $\rho(X, Y, Z)$ is not algebraic, and may be defined as the following limit:

$$
\rho(X, Y, Z)=\frac{2 Z}{(1-X Z)\left(1-X^{-1} Z\right)} \lim _{t \rightarrow 1^{-}} \sum_{k=0}^{\infty}(-t)^{k} \prod_{m=1}^{k} \frac{\left(1-q^{m} Y Z\right)\left(1-q^{-m} Y^{-1} Z\right)}{\left(1-q^{-m} X Z\right)\left(1-q^{m} X^{-1} Z\right)}
$$

It would be interesting to investigate its singularity structure.

Finally, the connection to cluster algebra should also allow us to consider quantum versions of the T-system, via the quantum cluster algebra construction of [1]. A first step in this direction was taken in [5] for the case of the $A_{1}$ quantum T-system. Presumably, such quantum deformations should yield new non-trivial deformations of the Lambdadeterminant and hopefully enrich our understanding of ASMs.

\section{References}

[1] A. Berenstein, A. Zelevinsky, Quantum Cluster Algebras, Adv. Math. 195 (2005) 405-455. arXiv:math/0404446 [math.QA].

[2] D. Bressoud, Proofs and confirmations: The story of the alternating sign matrix conjecture, MAA Spectrum, Mathematical Association of America, Washington, DC (1999), 274 pages.

[3] P. Di Francesco, The solution of the $A_{r}$ T-system for arbitrary boundary, Elec. Jour. of Comb. Vol. 17(1) (2010) R89. arXiv:1002.4427 [math.CO].

[4] P. Di Francesco and R. Kedem, Q-systems as cluster algebras II. arXiv:0803.0362 [math.RT].

[5] P. Di Francesco and R. Kedem, The solution of the quantum $A_{1}$ T-system for arbitrary boundary, Comm. Math. Phys. 313, No 2, (2012) 329-350. arXiv:1102.5552 [math$\mathrm{ph}]$.

[6] P. Di Francesco and R. Kedem, T-systems with boundaries from network solutions, Elec. Jour. of Comb. Vol. 20(1) (2013) P3. arXiv:1208.4333 [math.CO].

[7] C. Dodgson, Condensation of determinants, Proceedings of the Royal Soc. of London 15 (1866) 150-155.

[8] N. Elkies, G. Kuperberg, M. Larsen and J. Propp, Alternating-Sign Matrices and Domino Tilings (Parts I and II) Jour. of Alg. Comb. Vol 1, No 2 (1992), 111-132 and Vol 1, No 3 (1992), 219-234.

[9] S. Fomin and A. Zelevinsky Cluster Algebras I. J. Amer. Math. Soc. 15 (2002), no. 2, 497-529. arXiv:math/0104151 [math.RT]. 
[10] G. Kuperberg, Another proof of the alternating-sign matrix conjecture, Int. Math. Res. Notices No 3 (1996) 139-150. arXiv:math/9712207.

[11] B. Lindström, On the vector representations of induced matroids, Bull. London Math. Soc. 5 (1973) 85-90.

[12] I. M. Gessel and X. Viennot, Binomial determinants, paths and hook formulae, Adv. Math. 58 (1985) 300-321.

[13] R. Pemantle and M.C. Wilson, Asymptotics of multivariate sequences. I. Smooth points of the singular variety, J. Combin. Theory Ser. A, 97(1) (2002) 129-161; Asymptotics of multivariate sequences, II. Multiple points of the singular variety, Combin. Probab. Comput. 13 (2004) 735-761; Twenty combinatorial examples of asymptotics derived from multivariate generating functions, SIAM Review, 50 (2008) 199-272.

[14] D. Robbins and H. Rumsey, Determinants and Alternating Sign Matrices, Advances in Math. 62 (1986) 169-184.

[15] D. Speyer, Perfect matchings and the octahedron recurrence, J. Algebraic Comb. 25 No 3 (2007) 309-348. arXiv:math/0402452 [math.CO]. 